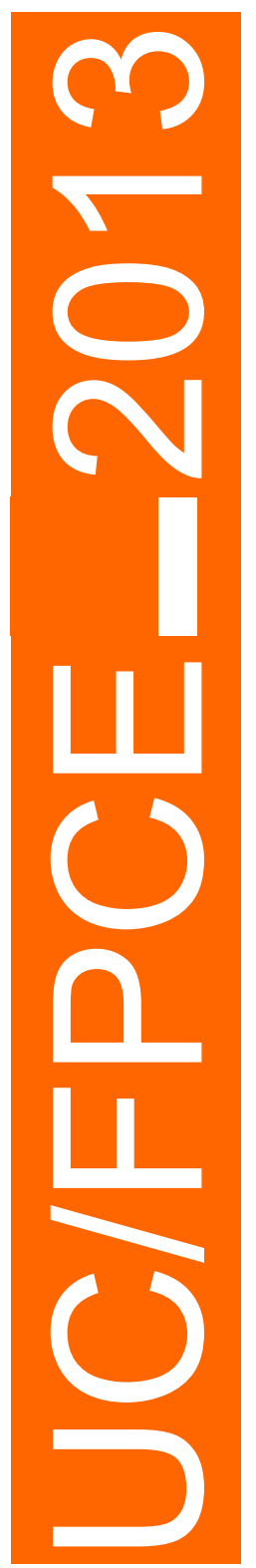

Universidade de Coimbra

Faculdade de Psicologia e de Ciências da Educação

Adult attachment and dyadic adjustment: The mediating role of shame

Teresa Maria Caldeira Martins (e-mail: tcmartins@netcabo.pt)

Dissertação de Mestrado em Psicologia Clínica e da Saúde, subárea de especialização em Intervenções Cognitivo-

Comportamentais nas Perturbações Psicológicas da Saúde, sob a orientação da Doutora Helena Moreira 


\section{Adult attachment and dyadic adjustment: The mediating role of shame}

Nas últimas décadas, o funcionamento das relações amorosas e a satisfação conjugal têm sido alvo de muitos estudos, nomeadamente numa tentativa de compreensão dos principais factores que podem contribuir para a qualidade dos relacionamentos amorosos. A problemática da satisfação e qualidade conjugal assume cada vez maior relevância devido ao aumento progressivo das taxas de divórcio, que se tem também tornado mais precoce. Compreender o que pode comprometer o funcionamento diádico é importante para o desenho de estratégias de intervenção eficazes que conduzam a uma maior satisfação conjugal e qualidade dos relacionamentos amorosos.

A maior parte dos estudos desenvolvidos neste contexto usou a teoria da vinculação como quadro conceptual. Esta teoria tenta explicar o modo como formas mais ou menos saudáveis de relações amorosas surgem como adaptações razoáveis a experiências sociais precoces. Mas se a relação entre a vinculação e a qualidade dos relacionamentos amorosos está já bem documentada, os mecanismos envolvidos continuam por esclarecer. Estes podem incluir variáveis individuais que são moldadas precocemente na interacção com figuras de vinculação e que afectem o funcionamento diádico, como é o caso da vergonha. No entanto, esta questão nunca foi investigada. Desta forma, o objectivo deste trabalho foi investigar o possível papel mediador da vergonha externa e interna na relação entre a vinculação do adulto e o ajustamento diádico.

Para tal, foi desenvolvida uma bateria de instrumentos de auto-resposta para medir a vinculação do adulto, a vergonha externa e interna e $o$ ajustamento diádico, e um questionário sócio-demográfico, que foram disponibilizados na internet. Estes foram acompanhados por um texto introdutório em que foram explicitadas todas as questões éticas, nomeadamente as garantias de confidencialidade e anonimato dos dados. A divulgação do trabalho e o convite para participação no mesmo foram feitos por correio electrónico. Através deste procedimento foi recolhida uma amostra composta por 228 sujeitos, maiores de 18 anos e envolvidos numa 
relação amorosa há pelo menos seis meses. Os dados obtidos foram sujeitos a análise estatística usando o programa Statistical Package for the Social Sciences (SPSS, versão 20.0) e a ferramenta informática PROCESS para análises de mediação (modelo de mediação 6 - mediação múltipla em série com duas variáveis mediadoras).

Os resultados mostraram que níveis mais elevados de ansiedade e evitamento de vinculação estão associados a um pior ajustamento diádico através de níveis elevados de vergonha externa, que, por sua vez, está associada a uma elevada vergonha interna. Adicionalmente, observou-se que a vergonha interna pode actuar, de forma isolada, como mediador desta relação, o que já não acontece no caso da vergonha externa.

Estes resultados salientam, então, a vergonha, particularmente, a vergonha interna, como um importante mediador da associação entre a vinculação do adulto e o ajustamento diádico. Estes dados são importantes não apenas pelo seu carácter inovador, mas também porque se identifica um processo psicológico que pode ser trabalhado com sucesso em contexto terapêutico e que é mais manejável do que os esquemas relacionais do indivíduo, nomeadamente os modelos dinâmicos internos.

Ajudar o indivíduo a lidar com a vergonha de forma mais adaptativa poderá contribuir para um melhor funcionamento interpessoal e para uma vivência mais gratificante das relações amorosas e, desta forma, promover uma revisão dos esquemas interpessoais.

Palavras chave: Vinculação; ajustamento diádico; vergonha interna; vergonha interna. 


\section{Adult attachment and dyadic adjustment: The mediating role of shame}

During the last decades, romantic relationships and marital functioning have been the target of many studies, namely in the attempt to clarify the main factors contributing to relationship quality. Intimate relationship functioning is gaining increasing scientific attention due to progressive increases in divorce rates, which is also occurring at earlier marriage times. Understanding of the factors that may compromise intimate relationships is critically important for the design of intervention strategies aimed at promoting a higher marital satisfaction and relationship quality. The majority of the studies has used the attachment theory as the conceptual framework, as it is believed that the enduring quality of attachment is a critical underlying feature of adult relationships and capacity for intimacy. Although the association between adult attachment and intimate relationship functioning is already well established, the mechanisms involved are still poorly understood. These may include individual factors that are shaped in early interactions with attachment figures and also impact dyadic functioning, such as shame. However, this issue has never been addressed. The present study aimed at investigating the mediating role of external and internal shame on the association between adult attachment and dyadic adjustment.

For that, a battery of self-report measures of adult attachment, external and internal shame and dyadic adjustment, and a socio-demographic questionnaire were developed and made available online. This was accompanied by an introductory text in which all ethical issues and information regarding confidentiality were explained. An invitation for the participation in this study was spread by e-mail. This procedure allowed for the collection of a sample composed by 228 individuals, older than 18 years old and committed to a romantic relationship for at least six months. The data analyses were conducted using the Statistical Package for the Social Sciences (SPSS, version 20.0) and the computational tool for path-analysis based mediation analysis, PROCESS (model 6 - serial multiple mediation 
model with two mediator variables).

The results showed that increased levels of attachment anxiety and avoidance are associated with a poorer dyadic adjustment, through higher levels of external shame, which is associated with high internal shame. In addition, the data demonstrated that internal shame alone may act as a mediator of the association between adult attachment and dyadic adjustment, which does not hold true for external shame.

These findings highlight the importance of shame, particularly internal shame, as a mediator of the association between adult attachment and dyadic adjustment. Besides being an innovative contribution, these data are important because they allowed the identification of a psychological process that may be successfully targeted in therapy and is easier to handle than relational schemata, namely the internal working models.

Helping individuals to deal with shame in a more adaptive fashion may contribute to a better interpersonal functioning and to a more gratifying experience of romantic relationships, which may promote revision of interpersonal schemata.

Key Words: Attachment; dyadic adjustment; internal shame; external shame. 


\section{Agradecimentos}

À Professora Cristina Canavarro por ter apostado em mim, por todos os ensinamentos, disponibilidade e preocupação. Por me ter desafiado para a sua equipa.

À Doutora Helena Moreira pela sua cuidadosa orientação e discussão pertinente e pela sua disponibilidade e compreensão.

A todos aqueles que colaboraram voluntariamente neste trabalho.

Aos meus pais, que me apoiaram nesta decisão que tomei num momento tão difícil da minha vida. Obrigada por acreditarem em mim e pela vossa compreensão e apoio nestes cinco anos.

Às minhas sobrinhas, que, durante estes anos, tiveram que partilhar a sua "Titi” com a Psicologia. Nem sempre esta divisão foi equilibrada...

À minha família, em geral, e aos meus amigos pelo tempo que não passei com eles... Obrigada pela vossa compreensão e por não terem desistido de mim! 


\section{Índice}

Enquadramento teórico 1

Introdução 1

Vinculação e relações amorosas 2

Mecanismos envolvidos na relação entre vinculação e a satisfação e qualidade relacional: O possível papel 6 da vergonha

O presente estudo 13

Bibliografia 14

$\begin{array}{ll}\text { Abstract } & 17\end{array}$

Introduction $\quad 17$

Methodology 22

Participants 22

Procedure 23

Measures $\quad 23$

Data analysis $\quad 24$

Results 25

Descriptive statistics and correlations 25

Mediation analysis 26

Discussion 28

References $\quad 35$

Conclusões 43

Bibliografia 45 


\section{Enquadramento Teórico}

\section{Introdução}

Encontrar alguém para partilhar a vida no contexto de uma relação estável e duradoura é um processo social chave que a maior parte dos adultos deseja concretizar. As relações amorosas detêm um papel central na vida dos adultos e estão associadas a uma série de resultados positivos, como o bemestar físico e psicológico. As relações amorosas de qualidade protegem os indivíduos da solidão, permitindo-lhes também alargar os seus horizontes em termos sociais, com um alargamento da sua rede de suporte social (Merz \& Jak, 2013). A problemática da satisfação e qualidade conjugal assume cada vez maior relevância devido ao aumento progressivo das taxas de divórcio, que se tem também tornado mais precoce. Compreender o que pode comprometer o funcionamento diádico é importante para o desenho de estratégias de intervenção eficazes que conduzam a uma maior satisfação conjugal e qualidade dos relacionamentos amorosos.

Nas últimas décadas, o funcionamento das relações amorosas e a satisfação conjugal têm sido alvo de muitos estudos, nomeadamente numa tentativa de compreensão dos principais factores que podem contribuir para a qualidade dos relacionamentos amorosos. Muitos destes estudos foram desenvolvidos à luz da teoria da vinculação. De facto, embora esta teoria tenha sido inicialmente desenvolvida para explicar a natureza complexa das relações entre as crianças e os cuidadores primários, foi mais tarde estendida a outros tipos de relações, nomeadamente à compreensão das ligações emocionais entre parceiros amorosos (e.g. Hazan \& Shaver, 1987). A teoria da vinculação constitui um enquadramento teórico para a conceptualização do modo como formas mais ou menos saudáveis de relações amorosas surgem como adaptações razoáveis a experiências sociais precoces (Treboux, Crowell, \& Waters, 2004). Acredita-se que as orientações de vinculação são mantidas na idade adulta, servindo como moldes para relacionamentos românticos seguros ou inseguros (Stackert \& Bursik, 2003). Mas se a relação entre a vinculação e a qualidade dos relacionamentos amorosos está já bem documentada, os mecanismos envolvidos continuam por esclarecer. Estes podem incluir variáveis individuais que são moldadas precocemente na interacção com figuras 
de vinculação, como é o caso da vergonha. O objectivo deste trabalho foi investigar o possível papel mediador da vergonha, interna e externa, na relação entre a vinculação do adulto e o ajustamento diádico.

\section{Vinculação e relações amorosas}

A teoria da vinculação foca-se na forma como as relações com as figuras de vinculação primárias afectam o desenvolvimento do indivíduo, o seu funcionamento adaptativo e a sua capacidade para gerir situações indutoras de stress, segurança e bem-estar, de uma forma geral. O principal objectivo do sistema comportamental de vinculação será a promoção do sentimento de segurança, através do estabelecimento de uma relação de base segura com uma figura cuidadora. O sistema de vinculação fornece ao indivíduo uma estrutura organizativa que lhe permite pedir ajuda em caso de necessidade e reconhecer pedidos de ajuda e fornecer apoio. Espera-se, então, que diferenças individuais nos padrões de vinculação tenham um papel no desenvolvimento de outras relações ao longo da vida e, em particular, de relações amorosas, e na forma como os casais enfrentam os desafios que a vida lhes impõe (Treboux et al., 2004).

A teoria da vinculação tem as suas raízes em Bowlby (1969/1982), que mostrou a importância crítica da ligação entre a criança e os cuidadores primários para o desenvolvimento do indivíduo (Feeney \& Hohaus, 2001). A vinculação é uma ligação emocional de longa duração que a criança estabelece com o cuidador. A natureza e qualidade destas relações precoces são determinadas pela disponibilidade emocional e responsividade do cuidador às necessidades da criança. As crianças que apresentam uma vinculação segura percebem os seus cuidadores como sensíveis e disponíveis e utilizam-nos como uma base segura, a partir da qual exploram o ambiente circundante, e como porto seguro, ao qual retornam em momentos de desconforto. Crianças com uma vinculação insegura ambivalente/preocupada vêem o seu cuidador como inconsistentemente responsivo e manifestam níveis elevados de comportamentos de vinculação para manterem o contacto com os seus cuidadores. Por seu lado, crianças com uma vinculação evitante não procuram o contacto com o cuidador quando perturbadas, uma estratégia que lhes permite manter uma ligação a um cuidador que as rejeita. Finalmente, crianças com vinculação desorganizada tiveram experiências com cuidadores que 
demonstraram medo ou comportamentos assustadores, ou uma comunicação afectiva perturbada. Consequentemente, estas crianças apresentam comportamentos de vinculação bizarros, contraditórios e incoerentes (Brumariu \& Kerns, 2010; Stackert \& Bursik, 2003).

As relações de vinculação fornecem um molde que a criança usa como estrutura em que integra informação fragmentada sobre o próprio e sobre o mundo, organizando-a num todo com significado (Brumariu \& Kerns, 2010; Stackert \& Bursik, 2003). As diferenças individuais no comportamento precoce de base segura reflectem o conhecimento da criança e as suas expectativas em relação ao seu próprio comportamento e ao da figura cuidadora. À medida que as experiências da criança com os cuidadores se repetem, os comportamentos de vinculação são automatizados, deixando de haver necessidade de uma reavaliação activa ou consciente de cada situação. Com o desenvolvimento de capacidades representacionais, os indivíduos retiram abstracções e constroem representações das suas experiências de vinculação (modelos dinâmicos internos). Com o tempo, estas representações específicas acabam por se generalizar, dando origem a guiões cognitivos e crenças sobre a função e a utilização de relações de vinculação, a forma como estas relações funcionam e aquilo que o indivíduo pode obter destas relações (Treboux et al., 2004). Estas representações generalizadas servem como um filtro para a compreensão das experiências, orientando o comportamento do indivíduo em situações associadas a vinculação. Quanto mais coerente, informada e organizada for a representação, mais eficaz será o guião usado nas relações actuais e futuras (Treboux et al., 2004).

Acredita-se que as orientações de vinculação são relativamente estáveis e que irão influenciar as relações interpessoais dos indivíduos ao longo da vida (Berlin, Cassidy, \& Appleyard, 2008; Parade, Lerkes, \& Balnkson, 2010; Stackert \& Bursik, 2003). No entanto, também é aceite que estes modelos podem ser revistos com base em todo e qualquer relacionamento significativo e que os indivíduos podem desenvolver crenças específicas para determinadas relações com base em experiências reais com parceiros específicos (Mikulincer, Florian, Cowan \& Cowan, 2002; Treboux et al., 2004). O envolvimento numa relação romântica estável e satisfatória pode permitir a revisão dos modelos dinâmicos internos por indivíduos com vinculação insegura, que podem passar a adoptar estratégias de maior confiança no parceiro (Merz \& Jak, 2013). 
Tal como já referido, o amor romântico pode ser interpretado como um processo de vinculação, considerando-se o casal como a relação de vinculação protótipo da idade adulta. Este tipo de relação comporta uma série de comportamentos que se consideram característicos de uma relação de vinculação: o indivíduo (1) mantém proximidade ao parceiro, (2) recorre ao parceiro como porto seguro quando se sente ameaçado ou necessita de conforto (a função básica das relações amorosas é a regulação dos sentimentos de segurança do parceiro, o que, por sua vez, apresenta benefícios em termos de saúde física e psicológica); (3) usa o parceiro como uma base segura a partir da qual explora o mundo envolvente (os sentimentos de segurança assegurados pela relação de vinculação acarretam uma exploração aumentada, que vai influenciar o funcionamento dos parceiros fora da relação - e.g. a sua capacidade para satisfazer objectivos pessoais); e (4) experiencia desconforto em alturas de separação ou quando o parceiro não está disponível (Selcuk, Zayas, \& Hazan, 2010). Apesar das semelhanças entre a vinculação precoce e a vinculação do adulto, existem duas diferenças importantes, nomeadamente ao nível das transacções e dos sistemas comportamentais envolvidos. De facto, ao contrário da vinculação precoce, em que as transacções são feitas predominantemente num sentido (o cuidador satisfaz as necessidades da criança), na vinculação do adulto as transacções pautam-se pela reciprocidade; adicionalmente, este último tipo de vinculação, para além dos sistemas de vinculação e de prestação de cuidados, envolve também o sistema sexual, que assume importância desde o início dos relacionamentos amorosos, já que a atracção sexual é geralmente a motivação primária para a procura de proximidade ao parceiro. No entanto, com o tempo, a importância deste sistema na manutenção das ligações do casal diminui, e a vinculação e cuidados mútuos passam a assumir um papel mais central (Selcuk et al., 2010).

Hazan e Shaver (1987), propuseram a existência de diferentes padrões de vinculação no adulto que influenciam as formas como os adultos experienciam o amor romântico e fornecem uma estrutura para a regulação do comportamento nos relacionamentos amorosos, sendo preditivos de variadas dinâmicas relacionais. Actualmente, reconhece-se que a vinculação deve ser interpretada de forma dimensional, e não categorial, sendo reconhecidas duas dimensões de vinculação: a ansiedade e o evitamento. A ansiedade reflecte o grau em que as pessoas se preocupam com a disponibilidade do parceiro, ou com o seu apoio 
em alturas de necessidade. Uma elevada ansiedade de vinculação está associada a cuidados inconsistentes e não fiáveis na infância e a modelos dinâmicos internos incertos sobre o Eu e os Outros. Como tal, o sistema de vinculação permanece cronicamente activado (estratégia de hiperactivação), o que faz com que estes indivíduos sejam hipervigilantes para sinais de ameaça, ao mesmo tempo que anseiam e procuram uma ligação excessiva e conforto pelos outros. Os indivíduos com elevada ansiedade de vinculação desejam desesperadamente a aceitação e apoio dos outros, mas, devido à sua percepção de falta de valor pessoal, tendem a duvidar da sua disponibilidade e responsividade (Fraley \& Shaver, 2000; Joel, MacDonald, \& Shimotomai, 2011; Selcuk et al., 2010). O evitamento espelha o grau em que os indivíduos desconfiam das boas intenções dos outros e lutam por manter uma distância emocional e independência do parceiro relacional (Fraley \& Shaver, 2000; Mikulincer et al., 2002). Níveis elevados de evitamento estão associados a níveis baixos, mas estáveis, de apoio na infância, que fazem com que os indivíduos com uma vinculação evitante apresentem uma desactivação crónica do sistema de vinculação, bem como desconforto com a proximidade e dependência dos outros (Joel et al., 2011; Selcuk et al., 2010). Desta forma, estas dimensões, mais do que a presença ou ausência de uma ligação de vinculação, reflectem sobretudo diferenças nas estratégias emocionais e comportamentais que os indivíduos usam para lidar com a inacessibilidade percebida da figura de vinculação. Assim, indivíduos com uma vinculação segura têm facilidade em confiar e depender dos outros, e em deixar os outros dependerem de si, e envolvem-se em estratégias relacionais mais adaptativas e construtivas, vendo-se a si próprios como merecedores de amor e socialmente competentes, e aos outros como confiáveis e fiáveis. Como tal, não apresentam dificuldades em estabelecer e manter relações íntimas. Estes indivíduos descrevem as suas experiências conjugais e relacionais como felizes e de confiança e terão também mais facilidade para entrar neste tipo de relacionamentos, lidando de forma mais eficaz com os conflitos que surgem na relação. Em contraste, indivíduos com uma vinculação ansiosa caracterizam-se por um desejo forte de proximidade e protecção nas relações amorosas, mas apresentam ruminação crónica, preocupação e dúvida sobre a disponibilidade do parceiro romântico e medo de abandono, demonstrando uma maior labilidade emocional, uma maior frequência e gravidade de conflitos e níveis mais baixos de confiança. Estes indivíduos tendem a perceber variabilidade na 
acessibilidade e responsividade dos seus parceiros e tentam captar a sua atenção e desencadear cuidados através de uma intensificação de expressões que sinalizam o desejo de proximidade e procurando uma reafirmação constante do amor do outro. As suas relações são caracteristicamente obsessivas, envolvendo ciúme e uma atracção sexual extrema ao parceiro. Adicionalmente, tendem a comportar-se de forma negativa relativamente ao parceiro quando enfrentam situações indutoras de stress, por forma a tentarem manter a proximidade (e.g. expressão de tristeza ou de desconforto), já que a ostentação de calma e de recuperação é percebida como aumentando o risco de perder o contacto com um parceiro inconsistentemente acessível. Estes comportamentos podem, no entanto, ter um efeito paradoxal, dado que aumentam a insatisfação do parceiro com a relação, podendo conduzir, a longo prazo, a rejeição. Por sua vez, os indivíduos que têm uma vinculação evitante parecem evitar a proximidade e a dependência emocional: as suas relações caracterizam-se por desconforto com a proximidade, uma interdependência reduzida, forte preferência por distanciamento emocional e menor intimidade, auto-revelação e confiança, pois percebem o parceiro como consistentemente inacessível e irresponsivo. As suas experiências amorosas caracterizam-se por medo de intimidade. Desta forma, negam as suas próprias necessidades de vinculação, bem como as dos outros, o que faz com que se torne muito difícil para estas pessoas estabelecer relações genuinamente íntimas com os parceiros românticos (Joel et al., 2011; Merz \& Jak, 2013; Meyers \& Landsberger, 2002; Scott \& Cordova, 2002; Selcuk et al., 2010; Stackert \& Bursik, 2003).

\section{Mecanismos envolvidos na relação entre a vinculação e a satisfação e qualidade relacional: $O$ possível papel da vergonha}

Embora a relação entre os padrões de vinculação e a satisfação nas relações esteja já bem documentada, os mecanismos subjacentes a esta associação ainda não estão completamente esclarecidos. A maior parte dos estudos tem focado quer variáveis proximais, como a comunicação, a auto-revelação, a expressividade emocional, as interacções afiliativas e de controlo e as estratégias de coping, entre outras, quer factores distais, relativamente mais estáveis e globais, como o apoio social e o desconforto psicológico (revisto em Feeney, 2008; Meyers \& Landsberger, 2002). No entanto, pouca atenção tem sido dada a factores individuais, como é o caso da vergonha. De facto, a 
vergonha tem vindo a ser associada à satisfação e qualidade nos relacionamentos amorosos (Harper, 2011; Gross \& Hansen, 2000; Lutwak, Panish, \& Ferrari, 2003). Adicionalmente, este processo psicológico parece ter origem nas relações precoces com os cuidadores, estando relacionado com os padrões de vinculação primária. Por exemplo, observa-se que adultos com vinculação insegura (preocupada ou medrosa) apresentam elevada predisposição para a vergonha (Mills, 2005). Como tal, é possível que a vergonha possa estar envolvida nas vias que ligam a vinculação à satisfação e qualidade dos relacionamentos amorosos. No entanto, este aspecto nunca foi investigado.

Dado o enorme poder evolutivo dos relacionamentos interpessoais, e de forma a experimentarem sentimentos de segurança e de pertença, e a envolverem-se em papéis sociais vantajosos, os seres humanos são motivados a estimular o afecto positivo e a gerar imagens positivas de si nas mentes dos outros. Desta forma, desenvolveram capacidades cognitivas complexas que thes permitem monitorizar a sua atractividade na mente dos outros e fazer previsões sobre o que os outros pensam e sentem sobre si. A vergonha emerge como uma destas capacidades cognitivas, funcionando como um sinal de alarme de que existimos de forma negativa na mente dos outros, i.e., como não atraentes, sem valor ou defeituosos, e de que podemos ser rejeitados, excluídos, ignorados, magoados ou perseguidos (Matos, Pinto-Gouveia, \& Costa, 2013). Esta emoção auto-consciente tem vindo a ser associada à experiência interna do Eu como indesejável, não atraente, defeituoso, sem valor e sem poder (Gilbert, 2000).

A vergonha pode ter origem na relação da criança com os cuidadores primários. A predisposição para a vergonha pode ter origem em diferentes experiências precoces, nomeadamente em experiências de rejeição e em vinculação insegura. Schore (1994, 1996, cit. in Mills, 2005) chegou mesmo a afirmar que o estilo de vinculação da criança é essencialmente o seu estilo de regulação da vergonha, explicando que, quando as figuras parentais estão consistentemente inacessíveis, não há apoio para a regulação do afecto e a vergonha ajudará a criança a auto-regular-se por desligamento. De acordo com o mesmo autor, a indisponibilidade ou rejeição parental fomenta estratégias de retirada emocional e de exclusão de processamento de ordem superior de emoções que activam a necessidade de vinculação; a criança minimiza e inibe a expressão de emoções relacionadas com a vinculação e apresenta-se ansiosa, 
inibida e com predisposição para vergonha consciente ou sentida. Quando o comportamento das figuras de vinculação é inconsistente, interferindo com as tentativas da criança de se desligar, como forma de modular a sua activação, a criança não aprende a desligar-se e apresenta dificuldades em regular a sua activação, com elevada emocionalidade negativa (hostilidade, irritabilidade), impulsividade e vergonha "não sentida".

O desenvolvimento da vergonha ocorre de forma progressiva durante o desenvolvimento cognitivo e socialização, à medida que a criança vai adquirindo capacidade para avaliar experiências e estabelecer valores, padrões, regras, objectivos/metas e crenças relevantes para o seu auto-valor, se vai tornando mais auto-consciente e capaz de se auto-avaliar, e desenvolve novas competências de enfrentamento e resposta emocional. Adicionalmente, a criança tem que ser capaz de fazer atribuições sobre as causas dos acontecimentos e sobre a responsabilidade do Eu nos acontecimentos. À medida que a criança adquire capacidade para experienciar vergonha, a própria vergonha começa a condicionar o seu desenvolvimento, nomeadamente através da sua função de regulação interna: a vergonha foca a atenção no Eu, activa a auto-avaliação e contribui para o desenvolvimento de auto-conhecimento, o que vai influenciar a conduta moral e as relações interpessoais (Mills, 2005). Campos, Thein e Owen (2004, cit. in Mills, 2005) sugerem que a vergonha pode ser constituída por avaliações reflectidas comunicadas através de desapontamento, raiva, desaprovação, nojo ou desdém expresso pelas figuras cuidadoras e outras pessoas significativas. De forma semelhante, Lewis (1971, cit. in Mills, 2005) refere que a vergonha é precipitada por uma avaliação do Eu como um todo e que terá as suas raízes na necessidade de vinculação aos outros. A rejeição por um ente querido é uma experiência prototípica geradora de vergonha, uma vez que é muitas vezes percebida como uma rejeição global e incontrolável do Eu, devida a indesejabilidade pessoal (Lewis, 1971, cit. in Mills, 2005). Também Bowlby, em 1973 (cit. in Mills, 2005), salientava este aspecto na sua formulação da teoria da vinculação, quando sugeriu que os modelos dinâmicos internos do Eu são complementares aos das figuras de vinculação, explicando que uma criança que não é desejada pelos pais tende não só a sentir-se indesejada pelos pais, mas a acreditar que é essencialmente não desejável, não amável, que nunca será desejado ou amado por ninguém. De acordo com a teoria da vinculação, é possível que as memórias de vergonha, em 
que os cuidadores primários envergonharam o $\mathrm{Eu}$, em que o indivíduo experienciou o Eu como defeituoso, não amável, sem valor e indesejável na mente dos cuidadores, determinem a formação de modelos dinâmicos internos negativos do $\mathrm{Eu}$ e dos Outros e se tornem parte integrante dos esquemas relacionais (Matos et al., 2013). Baldwin (1992, 1997, 2005; cit. in Matos et al., 2013) propôs que os modelos dinâmicos internos não só influenciam as previsões do comportamento dos outros e do próprio durante interacções sociais, mas que também vão moldar a base das avaliações e experiências do próprio sobre o próprio (self-to-self), que podem dar origem a vergonha. Com base na teoria do afecto, Kaufman (1985, 1989, cit. in Mills, 2005) propôs que a vergonha é desencadeada de forma interpessoal pelo desapontamento relativo a expectativas de mutualidade de resposta, ou por quebras nas "pontes interpessoais". O autor defende que, nos primeiros anos de vida, violações de expectativas de mutualidade e a expressão parental de raiva seriam experienciadas como rupturas e seriam indutoras de vergonha. Mais tarde, a vergonha seria activada por métodos de controlo na família, pelo grupo de pares, por práticas escolares envolvendo a humilhação/embaraço deliberado por exortação directa, desvalorização, desqualificação, humilhação, culpabilização ou desdém, bem como por pressões para se atingirem os padrões de desempenho ou de excelência impostos pelos outros, e por expressões de desapontamento em situações de fracasso. $\mathrm{Na}$ idade adulta, uma outra fonte importante de vergonha é o sentimento de falta de poder em qualquer domínio da vida que é essencial para a segurança do indivíduo (e.g. relações, trabalho, saúde, vida familiar).

Kaufman (1985, 1989, cit. in Mills, 2005) defende que, ao longo do desenvolvimento, a vergonha é internalizada, através do armazenamento na memória de imagens de experiências repetidas associadas a vergonha, que incluem crenças derivadas das mensagens transmitidas no acontecimento original (e.g. auto-avaliações), imagens dos padrões de interaç̧ão (um guião do acontecimento que engloba o comportamento do próprio e dos outros e as causas e consequências da interacção), e imagens do "outro internalizado" (a figura parental, geralmente na forma de voz interior); e fica associada a afectos (e.g. experienciar a raiva como geradora de vergonha), a impulsos fisiológicos (e.g. experienciar a sexualidade como fonte de vergonha), ou a necessidades interpessoais inatas (vergonha com base em necessidade de relacionamentos, 
toque/carinho, identificação, diferenciação, afirmação, poder, etc). Depois de internalizada, a vergonha pode ser activada internamente, com base nas memórias de vergonha, e torna-se possível que os sentimentos e pensamentos de vergonha desencadeiem novos pensamentos/sentimentos de vergonha, numa espiral interna de vergonha que a reforça e a estende a outras partes do $\mathrm{Eu}, \mathrm{o}$ que faz com que a vergonha passe a ser sentida como um sentimento profundo de defeito.

As experiências relacionais passadas podem, portanto, ser codificadas como memórias interpessoais, que funcionam como lentes que guiam o processamento emocional e as interacções, momento a momento (Matos et al., 2013). Matos e Pinto-Gouveia (2010) mostraram que as memórias de vergonha podem funcionar como memórias traumáticas que se podem tornar centrais para a identidade e história de vida do indivíduo, conferindo-lhes significado e continuidade, e influenciando o seu comportamento e objectivos. Estas memórias de vergonha podem também moldar os modelos dinâmicos internos do Eu (como sem valor e indesejável) e dos Outros (como ameaçadores, duros, rejeitantes, críticos, hostis, etc). Como tal, as memórias de vergonha podem integrar os esquemas interpessoais do indivíduo e, desta forma, guiar as suas expectativas relativas à forma como é visto pelos outros e a resposta que irá obter deles, constituindo também a base das avaliação e experiências do próprio sobre o próprio (Matos et al., 2013).

A vergonha é uma emoção que assume uma importância crucial nas interacções sociais. De acordo com Gilbert (2000), as ameaças que dão origem a vergonha e desencadeiam estratégias submissas estão geralmente associadas à perda de aprovação e aceitação pelos outros, o que se explica pela evolução do ranking social e relações sociais humanas em torno do desejo de se ser atraente para os outros e de criar determinados tipos de impressão favorável nos outros. A percepção de que se possuem traços valorizados pelos outros é crucial para o sentido de auto-valor e auto-estima dos indivíduos (Gilbert, 2000). Como tal, a vergonha tem importantes implicações para a auto-identidade, surgindo como uma forma de afecto defensivo relacionado com a manutenção do estatuto/rank social, prevenção da rejeição pelos outros e negociação de estruturas sociais complexas (Matos, Pinto-Gouveia, \& Duarte, 2012).

A vergonha pode estar relacionada com uma experiência emocional momentânea, ou seja, um sentimento agudo e transitório (vergonha-estado), ou 
com uma predisposição para sentir vergonha (vergonha-traço; Matos et al., 2012). A predisposição para a vergonha compreende duas dimensões distintas: a vergonha interna e a vergonha externa, que se diferenciam com base no foco da atenção, pensamentos e comportamentos. No que respeita à vergonha externa, a atenção é dirigida para o exterior, para o mundo social externo, e está associada a avaliações globais negativas (de que o Eu existe de forma negativa na mente dos outros), de forma a que o comportamento do indivíduo se orienta no sentido de tentar influenciar de forma positiva a sua imagem na mente dos outros, ou de estratégias defensivas, como a fuga, a submissão ou o apaziguamento (Matos et al., 2012). A vergonha externa é pautada por pensamentos e sentimentos de que os outros vêem o Eu de forma negativa (i.e. com sentimentos de raiva ou desprezo) e/ou de que o Eu é visto como tendo características que tornam o indivíduo não atraente para os outros e, como tal, rejeitável ou vulnerável ao ataque dos outros (Gilbert \& Procter, 2006). Desta forma, pode constituir uma experiência social dolorosa e tornar inseguro o mundo social, activando uma série de defesas como o desejo de se esconder, mascarar ou não se ser visto, e ter um poderoso efeito inibitório no processamento de informação, o que faz com que o indivíduo sinta a sua mente ficar confusa ou em branco (Gilbert \& Procter, 2006). Adicionalmente, o indivíduo pode envolver-se numa série de processos de regulação emocional maladaptativos, como ruminação, supressão de pensamentos desagradáveis e dissociação (Matos et al., 2013). Por sua vez, a vergonha interna surge com o desenvolvimento da auto-consciência. Na vergonha interna, a atenção e o processamento cognitivo estão dirigidos para o interior, para as emoções, atributos pessoais e comportamento do Eu, focando-se nas suas deficiências, inadequações e falhas. A vergonha interna está mais relacionada com uma proximidade a um Eu indesejado do que com a distância a um Eu desejado, e está tipicamente associada a experiências internas dolorosas de auto-avaliações e julgamentos negativos e auto-criticismo. Desta forma, o sofrimento associado à vergonha interna é auto-persecutório (Gilbert, 2000; Matos et al., 2012). As experiências de vergonha envolvem, geralmente, uma interacção entre vergonha interna e externa, na qual uma potencia a outra, ou seja, a dor derivada do reconhecimento da baixa atractividade social do Eu acompanha uma autodesvalorização dura e auto-culpabilização. Num episódio de vergonha, o indivíduo experiencia o mundo externo a voltar-se contra si, sendo que a sua 
própria auto-avaliação e sentido de identidade (mundo interno) também se tornam críticos, hostis e persecutórios (Gilbert \& Procter, 2006). Considera-se pouco provável que o afecto doloroso da depreciação privada ocorra na ausência de consciência de que os outros partilham a mesma visão negativa do $\mathrm{Eu}$ (Matos et al., 2012). Gilbert (2010) propôs que as experiências negativas precoces com outros significativos, nomeadamente as experiências de vergonha, podem tornar o indivíduo vulnerável a vergonha externa, ou seja, sensível a pensamentos e sentimentos negativos sobre a forma como o Eu existe na mente dos outros. De acordo com o mesmo autor, a vergonha externa pode desencadear dois grandes tipos de defesa, ou estratégias de segurança: a vergonha interna e a humilhação. A vergonha interna leva o indivíduo a adoptar uma estratégia de subordinação ou submissão, que está associada a autodesvalorização, atribuições internas negativas, auto-monitorização e autoculpabilização. Por sua vez, a resposta de humilhação será uma resposta externalizante, em que o indivíduo adopta um comportamento dominante agressivo e de ataque, através do qual tenta criar um sentido de segurança pessoal por desvalorização dos outros, ou de ataque de indivíduos que possam constituir ameaça (Gilbert, 2010).

Portanto, a vergonha pode dirigir o comportamento em contextos sociais, influenciar os sentimentos sobre o próprio, moldar o sentido de auto-identidade e os sentimentos sobre a aceitação e desejabilidade social (Gilbert, 1998; Tangney \& Dearing, 2002). De acordo com Barrett (1995, 1998, cit. in Mills, 2005), a função adaptativa da vergonha será a de manter a aceitação dos outros e preservar a auto-estima, através da aprendizagem e manutenção de padrões sociais e da submissão aos outros. Desta forma, a vergonha terá três funções: regulação comportamental (reduzir a exposição a avaliação por distanciamento ou não envolvimento), regulação interna (focar a atenção em padrões sociais e atributos próprios) e regulação social (comunicar deferência aos outros). Com o objectivo de proteger o Eu e evitar a rejeição, o indivíduo pode desenvolver uma série de estratégias defensivas como raiva, nojo, desprezo, luta pela perfeição ou pelo poder, culpabilização, retirada, evitamento, humor, negação, esconder ou mascarar o Eu, a transferência de culpa, bem como o auto- e hetero-ataque (Fedewa, Burns, \& Gomez, 2005; Mills, 2005).

Dado que a vergonha pode ter origem na relação da criança com os cuidadores primários e que as memórias de vergonha podem também moldar os 
modelos operantes do Eu (como sem valor e indesejável) e dos Outros (como ameaçadores, duros, poderosos, hostis, críticos, rejeitantes, etc), é possível que a vergonha possa actuar como um mediador entre os padrões de vinculação e a satisfação nos relacionamentos amorosos. Neste contexto, há um trabalho que sugere haver uma associação entre a predisposição para a vergonha e o medo de intimidade (Lutwak et al., 2003). Por sua vez, MacDonald e Morley (2001) mostraram que a vergonha estava associada a não revelação das emoções devido ao medo das consequências da auto-revelação. Os padrões de comunicação nas relações também são influenciados pela vergonha, devido às estratégias defensivas adoptadas pelos indivíduos de forma a evitar que os outros percebam a sua vergonha (Harper, 2011). Claesson e Sohlberg (2002) demonstraram que indivíduos com elevados níveis de vergonha apresentavam uma maior probabilidade de virem a ser rejeitados ou abandonados em relações íntimas. Estes autores sugeriram que tal se deveria à preocupação excessiva destes indivíduos com a possibilidade de o seu Eu defeituoso ser descoberto e de serem rejeitados, e à dinâmica relacional associada que acaba por levar ao fim da relação. Finalmente, Gross e Hansen (2000) descreveram uma associação positiva entre a vergonha e o investimento nas relações amorosas, tendo sugerido que as pessoas que duvidam do seu valor próprio apresentam dificuldades interpessoais aumentadas, o que faz com que tenham uma maior percepção da importância destes relacionamentos. Estes autores propuseram também que indivíduos com necessidades aumentadas de amor e cuidados e com uma preocupação aumentada com a possibilidade de ofender ou magoar o parceiro podem ser mais sensíveis e vulneráveis a experiências de vergonha nas relações íntimas (Gross \& Hansen, 2000).

\section{O presente estudo}

Neste trabalho fomos investigar o possível papel mediador da vergonha, interna e externa, na relação entre a vinculação do adulto e o ajustamento diádico. A relevância deste estudo reside na ausência de trabalhos de investigação sobre esta questão e na necessidade de se identificarem mecanismos envolvidos na associação entre a vinculação do adulto e o funcionamento nas relações amorosas que sejam mais manejáveis do ponto de vista clínico do que os esquemas interpessoais, nomeadamente os modelos dinâmicos internos, por forma a promover um melhor funcionamento 
interpessoal e uma vivência mais gratificante das relações íntimas.

A presente dissertação apresenta-se no formato de artigo científico, tal como será submetido para publicação num periódico internacional com arbitragem científica.

\section{Bibliografia}

Berlin, L. J., Cassidy, J., \& Appleyard, K. (2008). The influence of early attachments on other relationships. In J. Cassidy \& P. R. Shaver (Eds.), Handbook of attachment: Theory, research and clinical applications $\left(2^{\text {nd }} \mathrm{Ed}\right.$.; pp. 333-347). New York: Guilford Press.

Brumariu, L. E., \& Kerns, K. A. (2010). Mother-child attachment patterns and different types of anxiety symptoms: Is there specificity of relations? Child Psychiatry and Human Development, 41, 663-674.

Claesson, K., \& Sohlberg, S. (2002). Shame and interpersonal scripts: Internalized and other scripts characterized by indifference, abandonment and rejection: Replicated findings. Clinical Psychology \& Psychotherapy, 59, 277 284.

Fedewa, B. A., Burns, L. R., \& Gomez, A. A. (2005). Positive and negative perfectionism and the shame/guilt distinction: Adaptive and maladaptive characteristics. Personality and Individual Differences, 38, 16091619.

Feeney, J. A. (2008). Adult romantic attachment: Developments in the study of couple relationships. In J. Cassidy \& P. R. Shaver (Eds.), Handbook of attachment: Theory, research and clinical applications ( ${ }^{\text {nd }}$ Ed.; pp. 456-481). New York: Guilford Press.

Feeney, J. A., \& Hohaus, L. (2001). Attachment and spousal caregiving. Personal Relationships, 8, 21-39.

Fraley, R. C., \& Shaver, P. R. (2000). Adult romantic attachment: Theoretical developments, emerging controversies, and unanswered questions. Review of General Psychology, 4, 132-154.

Gilbert, P. (1998). What is shame? Some core issues and controversies. In P. Gilbert, \& B. Andrews (Eds.), Shame: Interpersonal behaviour, psychopathology and culture (pp. 3-36). New York: Oxford University Press.

Gilbert, P. (2000). The relationship of shame, social anxiety and depression: The role of the evaluation of social rank. Clinical Psychology and 
Psychotherapy, 7, 174-189.

Gilbert, P. (2010). Compassion focused therapy. W. Dryden (Ed.). New York: Routledge.

Gilbert, P., \& Procter, S. (2006). Compassionate mind training for people with high shame and self-criticism: Overview and pilot study of a group therapy approach. Clinical Psychology and Psychotherapy, 13, 353-379.

Gross, C. A., \& Hansen, N. E. (2000). Clarifying the experience of shame: The role of attachment style, gender, and investment in relatedness. Personality and Individual Differences, 28, 897-907.

Harper, J. M. (2011). Regulating and coping with shame. In R. Trnka, K. Balcar, \& M. Kuška (Eds.). Re-constructing emotional spaces: From experience to regulation (pp. 189-206). Prague: Prague College of Psychosocial Studies Press.

Hazan, C., \& Shaver, P. (1987). Romantic love conceptualized as an attachment process. Journal of Personality and Social Psychology, 52, 511-524.

Joel, S., MacDonald, G., \& Shimotomai, A. (2011). Conflicting pressures on romantic relationships commitment for anxiously attached individuals. Journal of Personality, 79, 51-73.

Lutwak, N., Panish, J., \& Ferrari, J. (2003). Shame and guilt: Characterological vs. behavioral self-blame and their relationship to fear of intimacy. Personality and Individual Differences, 35, 909-916.

MacDonald, J, \& Morley, I. (2001). Shame and non-disclosure: A study of the emotional isolation of people referred for psychotherapy. British Journal of Medical Psychology, 74, 1-21.

Matos, M., \& Pinto-Gouveia, J. (2010). Shame as a traumatic memory. Clinical Psychology and Psychotherapy, 17, 299-312.

Matos, M., Pinto-Gouveia, J., \& Costa, V. (2013). Understanding the importance of attachment in shame traumatic memory relation to depression: The impact of emotion regulation processes. Clinical Psychology and Psychotherapy, 20, 149-165.

Matos, M., Pinto-Gouveia, J., \& Duarte, C. (2012). When I don't like myself: Portuguese version of the Internalized Shame Scale. The Spanish Journal of Psychology, 15, 1411-1423.

Merz, E.-M., \& Jak, S. (2013). The long reach of childhood: Childhood experiences influence close relationships and loneliness across life. Advances in 
Life Course Research. doi:1016/j.alcr.2013.05.002

Meyers, S. A., \& Landsberger, S. A. (2002). Direct and indirect pathways between adult attachment style and marital satisfaction. Personal Relationships, 9, 159-172.

Mikulincer, M., Florian, V., Cowan, P. A., \& Cowan, C. P. (2002). Attachment security in couple relationships: A systemic model and its implications for family dynamics. Family Process, 41, 405-434.

Mills, R. S. L. (2005). Taking stock of the deveopmental literature on shame. Developmental Review, 25, 26-63.

Parade, S. H., Lerkes, E. M., \& Blankson, A. N. (2010). Attachment to parents, social anxiety, and close relationships of female students over the transition to college. Journal of Youth and Adolescence, 39, 127-137.

Scott, R. L., \& Cordova, J. V. (2002). The influence of adult attachment styles on the association between marital adjustment and depressive symptoms. Journal of Family Psychology, 16, 199-208.

Selcuk, E., Zayas, V., \& Hazan, C. (2010). Beyond satisfaction: The role of attachment in marital functioning. Journal of Family Theory \& Review, 2, 258-279.

Stackert, R. A., \& Bursik, K. (2003). Why am I unsatisfied? Adult attachment style, gendered irrational relationship beliefs, and young adult romantic relationship satisfaction. Personality and Individual Differences, 34, 1419-1429.

Tangney, J., \& Dearing, R. (2002). Shame and guilt. New York: Guilford Press.

Treboux, D., Crowell, J. A., \& Waters, E. (2004). When "new" meets "old": Configurations of adult attachment representations and their implications for marital functioning. Developmental Psychology, 40, 295-314. 


\begin{abstract}
During the last decades, intimate relationship functioning has been the target of many studies, namely in the attempt to clarify the main factors contributing to relationship quality. The majority of these studies has used the attachment theory as the conceptual framework. It is well established that adult attachment is associated with intimate relationship quality. Nevertheless, the mechanisms involved are still poorly understood. These may include individual factors that are shaped in early interactions with attachment figures and impact romantic relationship functioning, such as shame. However, this issue has never been addressed. The present study aimed at investigating the mediating role of external and internal shame on the association between attachment and dyadic adjustment. The sample was composed by 228 individuals, who completed an online battery of self-report measures of adult attachment, dyadic adjustment, and external and internal shame. Testing of the hypothesised serial multiple mediation models showed that attachment anxiety and avoidance were associated with worst dyadic adjustment through high levels of external shame, which were associated with high internal shame. In addition, our results demonstrated that internal shame alone may mediate the association between attachment and dyadic adjustment. Our findings highlight shame as an important target to address in therapy in order to promote a better dyadic adjustment and relationship functioning. This work makes an innovative contribution by identifying a new mechanism that may underlie the link between adult attachment and intimate relationship functioning.
\end{abstract}

Key words: attachment, dyadic adjustment, internal shame, external shame.

\title{
Introduction
}

Romantic relationships and marital functioning have been extensively studied in the last years in the attempt to identify the main factors contributing to relationship quality (Meyers \& Landsberger, 2002; Overall \& Fletcher, 2010; Stackert \& Bursik, 2003). Attachment theory provides a conceptual framework for the understanding of psychological and context variables that may influence romantic relationship and marital functioning, as well as relationship maintenance and dissolution (Treboux, Crowell, \& Waters, 2004). Bowlby 
(1979) emphasized that the enduring quality of attachment is a critical underlying feature of adult relationships and capacity for intimacy. Indeed, although the attachment theory has been originally developed to explain the complex nature of the relationship between children and their primary caregivers, it was later extended to other types of interpersonal relationships, particularly the emotional bonds between adult romantic partners (e.g. Hazan \& Shaver, 1987). It is well established that the way adults think, feel or act in romantic relationships is influenced by their attachment styles (Berlin, Cassidy, \& Appleyard, 2008; Meyers \& Landsberger, 2002; Stackert \& Bursik, 2003), and that adult secure attachment is generally associated with greater relationship satisfaction and happier and more trustful experiences (McCarthy \& Maughan, 2010; Meyers \& Landsberger, 2002; Saavedra, Chapman, \& Rogge, 2010). It is currently accepted that attachment should be best interpreted in a dimensional way (Brennan, Clark, \& Shaver, 1998). Attachment anxiety is characterised by sensitivity to rejection and abandonment, reflects the extension in which people worry about the romantic partner's availability or his/her support in times of need, and is associated with clingy and dependent behaviour in relationships. In turn, attachment avoidance is characterised by discomfort with intimacy and closeness in relationships, mirrors the degree at which individuals distrust their partners' good intentions and strive to keep emotional distance and independence from their partners, and is associated with behaviours that reduce intimacy, such as emotional withdrawal (Fraley, Waller, \& Brennan, 2000; Joel, MacDonald, \& Shimotomai, 2011; Mikulincer, Florian, Cowan, \& Cowan, 2002; Pepping \& Halford, 2012; Selcuk, Zayas, \& Hazan, 2010). Attachment avoidance and anxiety are both negatively correlated with marital adjustment (e.g. Lopez, Riggs, Pollard, \& Hook, 2011). However, the mechanisms involved in the association between attachment and marital functioning are still poorly understood. The majority of the studies have focused on proximal relational variables, such as communication, self-disclosure, emotional expression, affiliative and control interactions and coping strategies, among others (reviewed in Meyers \& Landsberger, 2002). However, individual features are still poorly studied. One important individual variable that may impact intimate relationship functioning is shame, a self-conscious emotion that is shaped in early interactions with the primary caregivers. In adults, shame seems to be associated with a wide variety of psychological symptoms and maladjustment, 
such as anxiety (e.g. Fergus, Valentiner, McGrath, \& Jencius, 2010), depression (e.g. Gilbert, 2000), social anxiety (e.g. Gilbert, 2000; Matos, Pinto-Gouveia, \& Gilbert, 2013), fear of negative social evaluation, feelings of inferiority (Gilbert, McEwan, Bellew, Mills, \& Gale, 2009), and eating disorders (e.g. Troop, Allan, Serpell, \& Treasure, 2008), among others. Being essentially a social emotion, it is expected that shame will impact interpersonal interactions and intimate relationships.

Shame has a pivotal role in social interactions, having serious implications for self-identity. It is associated with the internal experience of the self as undesirable, unattractive, defective, unworthy and powerless (Gilbert, 2000). Shame may constitute a painful social experience, involving the perception of being evaluated by others as inferior, defective or unattractive (external shame), or may be internalised as a private feeling (internal shame), which is associated with negative self-evaluations and judgments focused on the self's flaws and shortcomings (Gilbert, 2000). Shame experiences may involve thoughts associated with themes so diverse as personal attributes, dependence/ independence, competition, sense of self, personal attractiveness, sexuality, issues of seeing and being seen, and wishes and fear of closeness (Nathanson, 1992). As such, shame may influence human functioning at individual, interpersonal, group and cultural levels (Tangney \& Dearing, 2002). Barrett (1995, 1998) proposed that an adaptive function of shame would be the maintenance of others' acceptance and self-esteem preservation, through learning and maintenance of social standards and submission to others. In turn, Nathanson (1992) suggested that one of shame's functions is to alert the individual for actions and attributes that may lead to rejection and to motivate efforts to avoid rejection. Consequently, the behavioural tendencies associated with shame include withdrawal, avoidance, hiding or masking the self, positive self-presentation, perfection striving, guilt transfer, denial, and attack of self and others. The experience of shame usually involves an interaction between internal and external shame (Gilbert \& Procter, 2006). Gilbert (2010) proposed that early negative experiences with the caregivers, namely experiences of shaming, would result in vulnerability to external shame, which could then activate two major defensive strategies, including an internalised shame response, associated with self-devaluation and negative internal attributions, and adoption of subordinate, submissive behaviour with self-monitoring and 
self-blame. According to this perspective, internal shame would have its origins in external shame. Then, each would amplify the other: the pain derived from the perception of the low social attractiveness of the self being associated with a harsh self-devaluation and self-blame (Gilbert \& Procter, 2006).

As stated by Lewis (1971), shame is rooted in the needs for attachment to others. Baldwin $(1992,1997,2005)$ proposed that the internal working models not only influence predictions about self and others' behaviours in social interactions, but also shape self-evaluation and self-to-self experiences that can generate shame. Schore $(1994,1996)$ argued that the child's attachment orientation is primarily a shame regulation style, and that when attachment figures are consistently unavailable and the child has no support in affect regulation, shame will help child's self-regulation through detachment and withdrawal. As a consequence, the child may minimize and inhibit the expression of attachment-related emotions and is likely to show anxiety, inhibition and proneness to shame (Schore, 1994, 1996). Lewis (1971) highlighted that rejection by a loved one is a prototypic shame-inducing experience, as it is usually perceived as a global and uncontrollable rejection of the self due to personal undesirability.

Thus, shame may drive behaviour in social interactions, influence one's feelings about oneself, and shape one's sense of self-identity and feelings of social desirability and acceptance (Gilbert, 1998; Tangney \& Dearing, 2002). Pinto-Gouveia and Matos (2011) have shown that shame memories are central elements of life narratives and self-identity, being perceived as reference marks for daily inferences and for future expectations. As shame may arise in early interactions with attachment figures (Consedine \& Magai, 2003; Mills, 2005; Pinto-Gouveia \& Matos, 2011), and shame memories may shape the internal working models of self (perceived as unworthy and undesirable) and others (perceived as threatening, harsh, powerful, rejecting, critical, hostile) and become part of relational schemata (Matos, Pinto-Gouveia, \& Costa, 2013), it is conceivable that shame may mediate the effects of adult attachment on romantic relationships and marital functioning. Indeed, a previous study demonstrated that individuals with high levels of shame are most likely to be abandoned and rejected in intimate relationships (Claesson \& Sohlberg, 2002). According to the authors, these individuals spend so much time worrying about whether their negative self will be discovered and about rejection that they set up relationship 
dynamics that ultimately lead to that outcome. In turn, MacDonald and Morley (2001) described an association between shame and non-disclosure of emotions, which was attributed to the anticipation of negative interpersonal responses to disclosure. Harper (2011) argued that couple communication may also be affected by shame-associated guardedness and by the strategies employed to prevent shame being discovered. Other studies showed positive associations between shame proneness and fears of intimacy (Lutwak, Panish, \& Ferrari, 2003) and between shame and relationship investment (Gross \& Hansen, 2000). Gross and Hansen (2000) argued that people that doubt their worth experience greater interpersonal difficulties, which makes them more aware of the importance of such relationships. They have also suggested that individuals with a higher need of love and care, and who have increased concerns about hurting or offending others in intimate relationships may be more sensitive and/or vulnerable to shaming experiences within relationships (Gross \& Hansen, 2000).

Being a social emotion that arises from early interactions with significant others, with impact on intimate relationships, it is possible that shame mediates the association between attachment and intimate relationship functioning. Nevertheless, to the best of our knowledge, this mechanism has never been investigated. Understanding the role of shame in this context may be important for the identification of new therapeutic targets, which may be easier to manage in therapy than interpersonal schemata. Thus, the present study aims at exploring the indirect effects of attachment anxiety and avoidance on dyadic adjustment, through external and internal shame. In this work, dyadic adjustment was measured through the composite assessment of dyadic consensus, satisfaction and cohesion. Dyadic adjustment is determined by the extension of problem dyadic differences, interpersonal tension and conflict, individual anxiety, dyadic satisfaction and cohesion, couple's consensus on issues that are important for relationship functioning (e.g. family, financial, affection and sexual issues), the quality of partners' interactions and shared activities, and global evaluation of the relationship (Busby, Christensen, Crane, \& Larson, 1995; Gomez \& Leal, 2008; Ward, Lundberg, Zabriskie, \& Barrett, 2009). It was expected that higher levels of attachment anxiety and avoidance would be associated with higher levels of external shame, which would be associated with high internal shame, which, in turn, would be associated with 
decreased dyadic adjustment.

\section{Methodology}

\section{Participants}

The sample comprised 228 subjects from the general population that met the following inclusion criteria: (a) being 18 years old or older; and (b) being in a romantic relationship for at least six months. The socio-demographic characteristics of the sample are presented in Table 1.

Table 1

Socio-demographic characteristics of the sample

\begin{tabular}{ll}
\hline & $\begin{array}{l}N=228 \\
\text { Mean }(S D) ; \text { observed range }\end{array}$ \\
\hline Age (years) & $33.77(9.98) ; 18-66$ \\
Relationship time (months) & $118.79(110.60) ; 6-588$ \\
Cohabiting time (months) & $75.34(106.16) ; 0-519$ \\
& $n(\%)$ \\
Gender & $62(27.2)$ \\
Male & $166(72.8)$ \\
Female & \\
Cohabitation status & $60(26.3)$ \\
Cohabiting & $168(73.7)$ \\
Non-cohabiting & \\
Education levels & $30(13)$ \\
Undergraduate studies & $109(47.8)$ \\
Graduate studies & $88(38.6)$ \\
Post-graduate studies & $1(0.4)$ \\
Missing information & $10(4.4)$ \\
Professional status & $0(0.0)$ \\
Employed & $25(11)$ \\
Unemployed & $7(3.1)$ \\
Retired & \\
Student & \\
Other & \\
Missing information & $(71.9)$ \\
& \\
&
\end{tabular}


Household income (per month)

$\begin{array}{ll}<800 € & 16(7) \\ 800-2000 € & 106(46.5) \\ 2000-3500 € & 75(32.9) \\ >3500 € & 26(11.4) \\ \text { Missing information } & 5(2.2)\end{array}$

\section{Procedure}

A battery of self-report questionnaires designed to measure adult attachment, internal and external shame and dyadic adjustment were provided to the participants online. This battery was accompanied by an introductory text where the inclusion criteria were stated and where information on the confidentiality of the data and ethical issues were explained. Only those people who agreed with these conditions filled out the questionnaire. An invitation for participation in this study was spread through e-mail.

\section{Measures}

Adult attachment. The Experiences in Close Relationships Relationship Structures (ECR-RS) scale (Fraley, Heffernan, Vicary, \& Brumbaugh, 2011) is a self-report instrument designed to measure attachment dimensions (anxiety and avoidance) in different close relationships (mother, father, romantic partner and best friend). It is also possible to obtain global measures of attachment anxiety and avoidance, through the estimation of the means of the two dimensions in the four relationship targets. For each target, the scale is composed by nine items rated in a 7-point Likert scale $(1=$ Strongly disagree; 7 = Strongly agree). Items 1-6 compose the avoidance subscale, while the anxiety scale is composed by items 7-9. Higher scores on these subscales are indicative of high attachment avoidance and anxiety. In this study, we used the global anxiety and avoidance scores. Cronbach's $\alpha$ for the Global Avoidance Subscale was .88, and for the Global Anxiety Subscale was .93.

Shame. The Internal Shame Scale (ISS; Cook, 1996; Portuguese version: Matos, Pinto-Gouveia, \& Duarte, 2012) is a self-report inventory comprising 30 items, 24 of which measure internal shame. These items are negatively worded 
and assess the frequency at which people experience feelings of shame. ISS also includes a self-esteem subscale, composed of six positively worded items, that was not used in the present study. All items are rated on a 5-point Likert scale $(0=$ Never to $4=$ Almost always $)$. Higher scores on the internal shame subscale reveal high internal shame. In this study, the Cronbach's $\alpha$ for the internal shame subscale was .95 .

The Other As Shamer Scale (OAS; Allan, Gilbert, \& Goss, 1994; Goss, Gilbert, \& Allan, 1994; Portuguese version: Lopes, Pinto-Gouveia, \& Castilho, 2005 ) is composed by 18 items measuring the frequency of feelings and experiences of external shame, rated on a 5 -point Likert scale $(0=$ Never to $4=$ Almost always). Higher scores on this scale are indicative of high external shame. In this study, the Cronbach's $\alpha$ was .91.

Relationship functioning. The Revised Dyadic Adjustment Scale (RDAS; Busby et al., 1995; Portuguese version: Pereira, Canavarro, \& Narciso, 2009) measures the adjustment in romantic relationships with regard to consensus, satisfaction and cohesion. It allows the evaluation of the perception that people have about couple's global dyadic adjustment. This measure is a self-report questionnaire consisting of 14 items rated in 5- or 6-point Likert scales. In this study, the internal consistency of the global scale was high (Cronbach's $\alpha=.82$ ).

\section{Data Analysis}

The data analyses were conducted using the Statistical Package for the Social Sciences (SPSS, version 20.0; IBM SPSS, Chicago, IL). Missing data were random and infrequent $(<5 \%)$ and were handled by simple group mean substitution. Socio-demographic data were not substituted.

Descriptive statistics were computed for all socio-demographic and study variables. Correlations between socio-demographic and study variables were performed to identify possible covariates. Cohen's guidelines (1988) were used for describing the effect sizes of reported correlations (i.e. small for correlations around .10 , medium for those near .30 , and large for correlations at .50 or higher). Significance was set at the .05 level.

Mediation models were tested through PROCESS (Hayes, 2013), a computational tool for path analysis-based moderation and mediation analysis. 
Using this SPSS macro, serial multiple mediation models ("model 6" in Hayes, 2013) with two mediator variables were estimated. A bootstrapping procedure, using 5000 resamples, was used to assess unconditional indirect effects. Bootstrapping is a nonparametric resampling procedure that is recommended for testing indirect effects, as it does not require the assumption of normality of the sampling distribution of the indirect effects (Hayes, 2013). This procedure creates $95 \%$ bias-corrected and accelerated confidence intervals (BCa CIs) of the indirect effects, with an indirect effect considered to be significantly different from zero if zero is not contained within the lower and upper CIs.

\section{Results}

\section{Descriptive Statistics and Correlations}

The means, standard deviations and intercorrelations for the study variables are presented in Table 2. With regard to attachment and dyadic adjustment, the results show that attachment avoidance and attachment anxiety were both negatively and moderately correlated with total dyadic adjustment ( $r$ $=-.29, p<.001$; and $r=-.30, p<.001$, respectively). That is, individuals high in attachment anxiety and avoidance present reduced dyadic adjustment. Attachment dimensions were also significantly correlated both to internal and external shame: there was a medium, positive correlation between anxiety and both internal $(r=.34, p<.001)$ and external shame $(r=.36, p<.001)$, and a medium to high, positive correlation between avoidance and internal $(r=.44, p$ $<.001)$ and external shame $(r=.41, p<.001)$. That is, individuals with high anxiety and avoidance in attachment relationships present more internal and external shame. The Pearson product-moment correlation coefficients showed that shame was moderately and negatively correlated with dyadic adjustment $(r$ $=-.42, p<.001$, for internal shame; and $r=-.34, p<.001$, for external shame). That is, higher shame levels are associated with poorer dyadic adjustment.

Bivariate associations between socio-demographic and study variables were also analysed. Significant correlations were found between gender $(0=$ male; $1=$ female $)$ and global attachment avoidance $(r=-.14, p=.038)$; education $(r=-.15, p=.025 ; 0=$ undergraduate studies; $1=$ graduate and postgraduate studies) and relationship duration $(r=.15, p=.027)$ and global attachment anxiety; and cohabiting time and global attachment anxiety $(r=.14$, 
$p=.031)$

Table 2

Descriptive statistics and correlations between socio-demographic and study variables and among study variables

\begin{tabular}{|c|c|c|c|c|c|c|}
\hline & Variables & $\begin{array}{c}\text { Mean }(S D) ; \\
\text { observed range }\end{array}$ & 1 & 2 & 3 & 4 \\
\hline \multirow[t]{2}{*}{1} & Attachment avoidance & $2.31(0.75)$ & - & & & \\
\hline & & $1-4.75$ & & & & \\
\hline \multirow[t]{2}{*}{2} & Attachment anxiety & $2.21(1.22)$ & $.38 * * *$ & - & & \\
\hline & & $1-6.33$ & & & & \\
\hline \multirow[t]{2}{*}{3} & Dyadic adjustment & $50.89(7.81)$ & $-.29 * * *$ & $-.30 * * *$ & - & \\
\hline & & $18-66$ & & & & \\
\hline \multirow[t]{2}{*}{4} & Internal shame & $25.93(15.32)$ & $.44 * * *$ & $.34 * * *$ & $-.42 * * *$ & - \\
\hline & & $0-88$ & & & & \\
\hline \multirow[t]{2}{*}{5} & External shame & $19.77(9.37)$ & $.41 * * *$ & $.36 * * *$ & $-.34 * * *$ & $.68 * * *$ \\
\hline & & $0-65$ & & & & \\
\hline
\end{tabular}

\section{Mediation Analysis}

To evaluate the indirect effect of attachment dimensions on dyadic adjustment through internal and external shame, serial multiple mediation models were estimated. In these models, dyadic adjustment acted as the dependent variable (DV), attachment dimensions (attachment anxiety or avoidance) as the independent variables (IV), and external and internal shame as the mediators $\left(\mathrm{M}_{1}\right.$ and $\mathrm{M}_{2}$, respectively). The effects of the IV on the proposed Ms (paths $a_{1}$ and $a_{2}$ ), the effects of $\mathrm{M}_{1}$ and $\mathrm{M}_{2}$ on the DV partialling out the effect of IV and the other M variable (paths $b_{1}$ and $b_{2}$ ), the direct effect of IV on DV after controlling for $\mathrm{M}_{1}$ and $\mathrm{M}_{2}$ (path $c^{\prime}$ ), and the total effect of IV on DV (path $c$ ) are presented in Figures 1 and 2. Gender, relationship duration and cohabiting time were entered as covariates.

Attachment anxiety (cf. Figure 1). A significant specific indirect effect of global attachment anxiety on dyadic adjustment through external and internal shame was found $\left(a_{1} \mathrm{~d}_{21} b_{2}=-.51\right.$, LLCI $=-.95$, ULCI $\left.=-.23\right)$, which means that 
people with increased attachment anxiety have a poorer dyadic adjustment as a result of higher levels of external shame, which in turn are associated with higher internal shame. A significant indirect effect was also found with regard to the association of attachment anxiety and dyadic adjustment though internal shame $\left(a_{2} b_{2}=-.21\right.$, LLCI $=-.49$, ULCI $\left.=-.003\right)$. The results show that external shame alone does not act as a mediator of the effects of global attachment anxiety on dyadic adjustment $\left(a_{1} b_{1}=-.17\right.$, LLCI $=-.64$, ULCI $\left.=.27\right)$. A significant direct effect of global attachment anxiety on dyadic adjustment was observed, partialling out internal and external shame $\left(c^{\prime}=-1.00, p=.02\right)$.

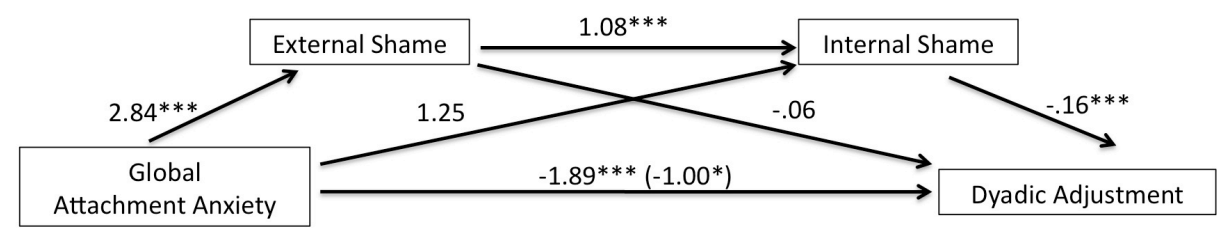

Figure 1. Statistical diagram of the serial multiple mediator model for the presumed shame influence on the effects of global attachment anxiety on dyadic adjustment. Path values represent unstandardised regression coefficients. The value in parentheses represents the total effect of attachment anxiety on dyadic adjustment before the inclusion of the mediating variables. The value in parentheses represents the direct effect, from bootstrapping analysis, of attachment anxiety on dyadic adjustment after inclusion of the mediators. ${ }^{*} \mathrm{p}<$ $.05 ; * * \mathrm{p}<.01 ; * * * \mathrm{p}<.001$.

Attachment avoidance (cf. Figure 2). A significant specific indirect effect of global attachment avoidance on dyadic adjustment through external and internal shame was observed $\left(a_{1} \mathrm{~d}_{21} b_{2}=-.83\right.$, LLCI $=-1.54$, ULCI $\left.=-.38\right)$, meaning that people with increased attachment avoidance present a worst dyadic adjustment as a result of higher levels of external shame, which, in turn, are associated with high internal shame. The data also demonstrate a significant indirect effect of attachment avoidance on dyadic adjustment through internal shame $\left(a_{2} b_{2}=-.63, \mathrm{LLCI}=-1.18, \mathrm{ULCI}=.28\right)$. The results show that external 
shame alone does not act as a mediator of the effects of global attachment avoidance on dyadic adjustment $\left(a_{1} b_{1}=-.39\right.$, LLCI $=-1.21$, ULCI $\left.=.34\right)$. No significant direct effect of global attachment avoidance on dyadic adjustment, controlling for internal and external shame, was observed $\left(c^{\prime}=-1.24, p=.09\right)$.

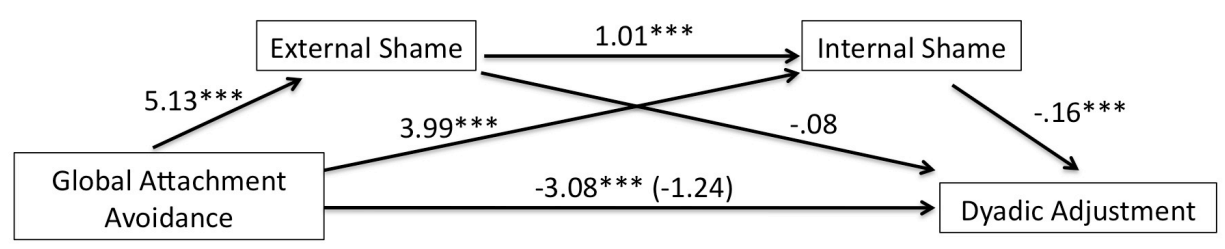

Figure 2. Statistical diagram of the serial multiple mediator model for the presumed shame influence on the effects of global attachment avoidance on dyadic adjustment. Path values represent unstandardised regression coefficients. The value in parentheses represents the total effect of attachment avoidance on dyadic adjustment before the inclusion of the mediating variables. The value in parentheses represents the direct effect, from bootstrapping analysis, of attachment avoidance on dyadic adjustment after inclusion of the mediators. *p $<.05 ; * * \mathrm{p}<.01 ; * * * \mathrm{p}<.001$.

\section{Discussion}

This study explored the mediating role of shame on the effects of attachment avoidance and anxiety on dyadic adjustment. As expected, the results showed that increased attachment anxiety and avoidance are associated with poorer dyadic adjustment through high levels of external shame, which are associated with high internal shame. In addition, results demonstrated that the association of attachment and dyadic adjustment may be mediated by internal shame alone. The data obtained in this work did not confirm the hypothesis of a mediator role for external shame alone on the association between attachment dimensions and dyadic adjustment.

As described in other studies (Bogaert \& Sadava, 2002; Lopez et al., 2011; Stackert \& Bursik, 2003), a significant negative association between attachment avoidance and anxiety and dyadic adjustment was observed. In 
general, attachment security (low anxiety and avoidance) is associated with positive beliefs about intimate relationships, the formation of more stable relationships, increased satisfaction with dating and marital relationships, higher levels of intimacy, commitment and emotional involvement within the relationship, and positive patterns of communication and interactions in close relationships (Mikulincer et al., 2002). It has been argued that these better outcomes may result from the affective consequences of secure attachment (reduced distress due to proximity to a significant one), which contribute to a positive orientation toward closeness and intimacy that, in turn, promote involvement in long-lasting romantic relationships (Mikulincer et al., 2002). In addition, low attachment anxiety and avoidance, which reflect positive internal working models of self and others (Feeney, 2008), may foster the development of a cognitive-affective framework for conflict management, thus contributing for the maintenance of satisfying relationships (Mikulincer et al., 2002; Selcuk et al., 2010). Finally, the sense of secure attachment may facilitate the satisfaction of other psychological needs, such as exploration, affiliation and caregiving, within the relationship. Again, this would increase relationship satisfaction (Feeney \& Hohaus, 2001; Mikulincer et al., 2002; Selcuk et al., 2010). In contrast, attachment avoidance is positively associated to divorce and multiple marriages, whereas attachment anxiety is correlated with staying in an unhappy marriage; and both dimensions are positively associated with destructive and coercive conflict management behaviours and negatively related to constructive and compromising behaviours (reviewed in Selcuk et al., 2010).

Our results have shown that both attachment dimensions were significantly and positively correlated with both internal and external shame. Although not directly comparable, our results somehow contrast the ones obtained by Gross and Hansen (2000) and Lopez et al. (1997), which showed that shame was positively related to fearful and preoccupied attachment, but failed to demonstrate a correlation to dismissing attachment (high in attachment avoidance). However, different shame and attachment measures and attachment conceptualization (dimensional) were used in this work. The herein presented results suggest that individuals high in attachment avoidance possess a negative view of the self, as reflected by their high levels of internal shame. In addition, the data is also indicative that the avoidant behaviour of these individuals in relationships, which is generally attributed to their negative view of significant 
others (as rejecting or unavailable), may also be due to their perception that others view them in a negative way (external shame). Thus, it is possible that individuals high in attachment avoidance perceive others as rejecting because they believe they exist negatively in their minds, which implies also evaluating themselves negatively (internal shame). Indeed, Goss et al. (1994) have shown that there is a high correlation between external and internal shame cognitions, which was also seen in the present study. Our data is congruent with Gross and Hansen (2000) hypothesis that dismissing individuals' minimization of others constitutes a defensive, self-protective strategy with an underlying negative sense of self. In line with this, several studies have reported that individuals with high attachment avoidance presented high levels of anxiety and somatisation responses on indirect measures, but low conscious access to anxiety-related memories and high levels of defensiveness (Mikulincer, Florian, \& Tolmacz, 1990; Mikulincer, Florian, \& Weller, 1993; Mikulincer \& Orbach, 1995).

Our results show that internal and external shame are negatively associated with dyadic adjustment. Our data is consistent with the observation that the way people believe they are evaluated by their partners plays a pivotal role in the functioning of intimate relationships (Overall \& Fletcher, 2010). Individuals who have a positive perception about their partner's regard report high relationship satisfaction, trust in their partners' continued regard and constructively cope with relationship problems, by trying to restore closeness (Campbell, Simpson, Kashy, \& Fletcher, 2001; Murray, Bellavia, Rose, \& Griffin, 2003). In turn, individuals that believe that they exist negatively in the mind of their partners experience poorer relationship quality and protect themselves from expected rejection by devaluing and withdrawing from their partners (Murray et al., 2003). Individuals high in internal shame, who feel inferior and defective, and devalue themselves, may be pessimistic about the likelihood of others accepting and valuing the self (Overall \& Fletcher, 2010) and may underestimate the positive view of their romantic partners (Murray, Holmes, Griffin, Bellavia, \& Rose, 2001), as may people high in external shame.

Shame may also impact dyadic adjustment through conflict management and interpersonal tension, as it activates feelings of unworthiness during conflicts, generating hostile defensiveness (Williamson, Sandage, \& Lee, 2007). 
In addition, it is associated with self-blaming (regarding one's behaviour and character) and blaming others (Lutwak et al., 2003), as well as attacking the self and others (Fedewa, Burns, \& Gomez, 2005), all of which may compromise the maintenance of supportive and satisfying close relationships. Indeed, self-blame constitutes a shame-associated defensive strategy, which is used in response to conflict in intimate relationships (Gilbert \& Procter, 2006). It is used by highshame women in abusive relationships, contributing to the permanence of these women in the relationships (Andrews \& Brewin, 1990; Buchbinder \& Eisikovits, 2003). Self-blaming and self-criticism arising during relationship conflicts may also promote both internal and external shame and increase the sense of internal threat (Castilho \& Pinto-Gouveia, 2011; Gilbert \& Irons, 2004; Gilbert \& Procter, 2006). Other behavioural strategies associated with shame may also affect dyadic consensus and relationship satisfaction. For instance, individuals may adopt a submissive posture, accepting all the partner's decisions and impositions, even in highly important issues, and not be able to express their opinions, feelings and needs. Indeed, shame operates through damage limitation strategies, including submissive behaviour (Gilbert, 2010). In a potential shaming-inducing situation, the individual may either try to escape or attempt to adopt submissive display and behaviours to limit possible attacks (Gilbert, 2000). In addition, shame may impact relationship functioning by increasing individual anxiety levels, namely social and generalized anxiety (Fergus et al., 2010; Gilbert, 2000; Hedman, Ström, Stünkel, \& Mörtberg, 2013).

Additional mechanisms by which shame may affect dyadic adjustment include fears of intimacy (Lutwak et al., 2003), as intimacy is a crucial feature of intimate relationship functioning, promoting involvement in long-lasting romantic relationships (Mikulincer et al., 2002); sexual functioning (Harper, 2011; Lombardi, 2007); self-disclosure, namely of emotions (Laurenceau, Feldman Barrett \& Pietromonaco, 1998; MacDonald \& Morley, 2001); and communication (Harper, 2011). It is also possible that people high in shame tend to negatively evaluate their behaviour in the relationship and make negative global, internal and stable attributions about the self, particularly in times of stress, as they do in other life domains (Mills, 2005). This may also reduce relationship satisfaction and dyadic adjustment. In addition, the fear of losing attractiveness in the minds of others associated with shame (Gilbert, 
2000) may undermine the ability of high-shame individuals to deal with the affiliative needs of the partner, as they would see people outside the relationship as more attractive and powerful competitors, and a threat for their romantic relationship. This may also have a negative impact on relationship satisfaction (Mikulincer et al, 2002).

As attachment experiences may constitute a source of shame and shame may impact intimate relationship functioning, the possible mediating role of shame on the effect of attachment anxiety and avoidance on dyadic adjustment was studied. Given that internal shame may arise as a defensive mechanism against external shame (Gilbert, 2010), a serial mediation analysis was performed. Our results showed that external and internal shame jointly mediate the association between attachment and dyadic adjustment. In particular, our data demonstrated that both external and internal shame mediated the effect of attachment anxiety on dyadic adjustment, with a direct effect of attachment anxiety on dyadic adjustment. Internal shame alone also acted as a mediator of this association, whereas external shame only acted as a mediator through its association with internal shame. These data is consistent with Gilbert's model of shame (2010), which states that insecure attachment, particularly attachment anxiety, is at the roots of beliefs that others do not value the self, but rather look down on the self (external shame; Gilbert et al., 2009). When the individual believes that others hold a negative view of the self, they may engage in different defensive strategies. One of these strategies is the internalization of shame, which leads individuals to experience the self as inferior, worthless, defective and undesirable (Gilbert, 2010). These feelings of inferiority and the perceptions that others do not value the self put on a constant pressure on insecurely attached individuals to find acceptance in the eyes of others and self, leading to striving to avoid inferiority (Gilbert et al., 2009). Indeed, intimate relationships are highly competitive social arenas in which people strive to win a valued place in the hearts and minds of others, while being aware that others are making judgments about the self in comparison to others. The more insecure and uncertain people feel about their qualities (negative working model of self), the more competitive their orientation to social relationships tends to be, and the more processing systems become focused on social comparison, concerns with what others think about the self and the need for assertive or submissive behaviour, or for insecurity striving (Gilbert et al., 2009). As already 
mentioned, striving to avoid unwanted inferiority and abandonment/rejection, as well as clingy behaviour, may have a paradoxical effect on relationships, as they may increase partner's dissatisfaction with the relationship and, in the long term, lead to rejection (Selcuk, et al., 2010). In contrast, people securely attached, who feel comfortable in social relationships, do not fear inferiority or mistakes, partly because they view others as accepting and helpful, rather than rejecting and shaming (Gilbert et al. 2009). For humans, the main threat that triggers shame is generally related to rejection, and loss of acceptance and approval (Gilbert, 2000). Thus, the behaviour of anxiously attached people in relationships may further promote their internal shame, which will negatively impact the quality of their intimate relationships, namely dyadic adjustment.

Our data also showed that external and internal shame jointly act as mediators of the effects of attachment avoidance on dyadic adjustment, even in the absence of a direct effect between these two variables. Also in this case, the indirect effects of shame may be related to the defensive strategies elicited to conceal a defective self and to avoid feelings of inferiority. Indeed, compensation, masking the self and positive self-presentation are defensive mechanisms commonly used to conceal shame that have a calming effect as long as the individual believes that others will not uncover their shame (Harper, 2011). Putting down or devaluing others increases one's sense of self and is also used to avoid feelings of inadequacy. Intimacy avoidance, emotional distancing and independence also constitute defensive mechanisms used by people high in attachment avoidance to conceal the defective self and prevent others to discover one's flaws and shortcomings. Other ways of creating distance from others and devaluing their importance is rage and contempt (Gardner \& Grofein, 2005), which are also defensive strategies to deal with external shame (Gilbert, 2010). Thus, fearing discovery, the avoidant individual distances his/herself from the intimate partner so that the anticipated discovery of shame and the feared abandonment and rejection by the romantic partner when their shame, or "true" self is discovered never takes place (Harper, 2011; Murray et al., 2003). However, these are all maladaptive responses that may contribute to the end of intimate relationships (Harper, 2011). Therefore, we now propose that the devaluating self view of people with high internal shame and their fear of being perceived as undesirable by others (external shame), which may arise from early experiences with attachment figures, may have a negative effect on 
dyadic adjustment in two ways: (1) by insecure striving and the adoption of a clingy and dependent behaviour; and/or (2) through minimization of the importance of others and distancing in romantic relationships as a defensive strategy against discovery of one's "true", defective self. In turn, perceived or real rejection episodes may further increase shame in these individuals.

A number of limitations of this study must be noted. The primary of these is the cross-sectional design of the study, which does not allow causal inferences. In addition, dyadic adjustment is a dynamic process rather than a static outcome. Thus, these two aspects could be better understood using a longitudinal design. Second, there was an imbalance between men and women participating in this study $(27.2 \%$ and $72.8 \%$, respectively). It would then be important to increase the number of men in future studies, even more as we have found a correlation between gender and attachment avoidance. Third, there was a disproportion in education levels $(86.4 \%$ of the sample was composed of people with graduate level studies or higher) and in people cohabiting (73.7\%) or not with romantic partners $(26.3 \%)$. Although no correlation was found between cohabiting status and study variables, future studies should address these issues. Fourth, the representativeness of our sample may be questioned due the divulgation strategy used (e-mail). This feature may account for the sample imbalance with regard to education levels, and for the increased socioeconomic level of the sample when compared to Portuguese national levels. Another potential limitation is the high correlation between internal and external shame, which may have masked some effects of the external shame. Although the two constructs are conceptually independent, they may not be statistically independent. Finally, we must mention the use of self-report instruments, which may not accurately reflect what people actually feel, think or do in real life situations and maybe prone to the social desirability effect. However, this effect could have been minimised by the online, anonymous filling of the questionnaires.

Despite these limitations a number of strengths can be highlighted. This research focused on intrapersonal variables that are relevant in this scientific domain but have been poorly explored until now. To our knowledge it is the first study that addresses the mediating role of shame on the association between attachment and intimate relationship functioning. Up to now, the majority of the studies have focused on direct relations between attachment and 
dyadic functioning, attachment and shame and shame and relationship quality. In addition, this study has scientific and practical implications. Intimate relationship functioning, namely marital quality and satisfaction, is gaining increasing scientific attention due to progressive increases in divorce rates, which is also occurring at earlier marriage times. Thus, the understanding of the factors that may compromise intimate relationships assumes a critical importance in designing intervention strategies aimed at promoting a higher marital satisfaction and relationship quality. Although adult attachment is clearly related to dyadic functioning, the mechanisms involved are still poorly understood. In addition, early experiences with primary caregivers are structured in interpersonal schemata (the internal working models of self and others) that, like any other core schema, are not easily accessible for intervention and are resistant to change. Thus, identification of variables that are easier to handle in therapy is of crucial importance. In this study, we have identified internal and external shame as mediators of the effect of attachment anxiety and avoidance on dyadic adjustment. Helping people dealing with shame, namely in a more self-compassionate way, may contribute to more satisfying, gratifying and fulfilling relationships, which in turn may conduce to changes in attachment and interpersonal schemata and more adaptive interpersonal functioning. Revision of the autobiographical meaning associated with shame memories may also lead to revaluation and restructuring of the patient's internal working models of self and others (Matos et al., 2013). This study also suggests that it may be important to evaluate shame in the context of couple therapy.

\section{References}

Allan, S., Gilbert, P., \& Goss, K. (1994). An exploration of shame measures: II. Psychopathology. Personality and Individual Differences, 3, 293 299.

Andrews, B., \& Brewin, C. R. (1990). Attributions of blame for marital violence: A study of antecedents and consequences. Journal of Family and Marriage, 52, 757-767.

Baldwin, M. W. (1992). Relational schemas and the processing of social information. Psychological Bulletin, 112, 461-484.

Baldwin, M. W. (1997). Relational schemas as a source of if-then selfinference procedures. Review of General Psychology, 1, 326-335. 
Baldwin, M. W. (Ed.) (2005). Interpersonal cognition. New York: Guilford Press.

Barrett, K. C. (1995). A functionalist approach to shame and guilt development. In J. P. Tangney \& K. W. Fischer (Eds.), Self-conscious emotions: The psychology of shame, guilt, embarrassment, and pride (pp. 2563). New York: Guilford Press.

Barrett, K. C. (1998). A functionalist perspective to the development of emotions. In M. F. Mascolo \& S. Griffin (Eds.). What develops in emotional development? (pp. 109-133). New York: Plenum.

Berlin, L. J., Cassidy, J., \& Appleyard, K. (2008). The influence of early attachments on other relationships. In J. Cassidy \& P. R. Shaver (Eds.), Handbook of attachment: Theory, research and clinical applications $\left(2^{\text {nd }} \mathrm{Ed}\right.$.; pp. 333-347). New York: Guilford Press.

Bogaert, A. F., \& Sadava, S. (2002). Adult attachment and sexual behavior. Personal Relationships, 9, 191-204.

Bowlby, J. (1979). The making and breaking of affectional bonds. London: Tavistock/Routledge.

Brennan, K. A., Clark, C. L., \& Shaver, P. R. (1998). Self-report measurement of adult attachment: An integrative overview. In J. A. Simpson \& W.S. Rholes (Eds.). Attachment theory and close relationships (pp. 46-76). New York: Guilford Press.

Buchbinder, E., \& Eisikovits, Z. (2003). Battered women's entrapment in shame: A phenomenological study. American Journal of Orthopsychiatry, 73, 355-366.

Busby, D. M., Christensen, C., Crane, D. R., \& Larson, J. H. (1995). A revision of the dyadic adjustment scale for use with distressed and nondistressed couples: construct hierarchy and multidimensional scales. Journal of Marital and Family Therapy, 21, 289-308.

Campbell, L., Simpson, J. A., Kashy, D. A., \& Fletcher, G. J. O. (2001). Ideal standards, the self, and flexibility of ideals in close relationships. Personality and Social Psychology Bulletin, 27, 447-462.

Castilho, P., \& Pinto-Gouveia, J. (2011). Auto-criticismo: Estudo de validação da versão portuguesa da Escala das Formas do Auto-criticismo e Auto-tranquilização (FSCRC) e da Escala das Funções do Auto-criticismo e Auto-ataque (FSCS). Psychologica, 54, 63-86. 
Claesson, K., \& Sohlberg, S. (2002). Shame and interpersonal scripts: Internalized and other scripts characterized by indifference, abandonment and rejection: Replicated findings. Clinical Psychology \& Psychotherapy, 59, 277284.

Cohen, J. (1988). Statistical power analysis for the behavioural sciences. Hillsdale: Erbaum.

Consedine, N. S., \& Magai, C. (2003). Attachment and emotion experience in later life: The view from emotions theory. Attachment and Human Development, 5, 165-187.

Cook, D. R. (1996). Emprirical studies of shame and guilt: The internalized shame scale. In D. L. Nathanson (Ed.). Knowing feeling: Affect, script, and psychotherapy (pp. 132-165). New York: W.W. Norton.

Fedewa, B. A., Burns, L. R., \& Gomez, A. A. (2005). Positive and negative perfectionism and the shame/guilt distinction: Adaptive and maladaptive characteristics. Personality and Individual Differences, 38, 16091619.

Feeney, J. A. (2008). Adult romantic attachment: Developments in the study of couple relationships. In J. Cassidy \& P. R. Shaver (Eds.), Handbook of attachment: Theory, research and clinical applications ( $2^{\text {nd }}$ Ed.; pp. 456-481). New York: Guilford Press.

Feeney, J. A., \& Hohaus, L. (2001). Attachment and spousal caregiving. Personal Relationships, 8, 21-39.

Fergus, T. A., Valentiner, D. P., McGrath, P. B., \& Jencius, S. (2010). Shame- and guilt-proneness: Relationships with anxiety disorder symptoms in a clinical sample. Journal of Anxiety Disorders, 24, 811-815.

Fraley, R. C., Heffernan, M. E., Vicary, A. M., \& Brumbaugh, C. C. (2011). The experiences in close relationships - relationship structures questionnaire: A method for assessing attachment orientations across relationships. Psychological Assessment, 23, 615-625.

Fraley, R. C., Waller, N. G., \& Brennan, K. A. (2000). An item response theory analysis of self-report measures of adult attachment. Journal of Personality and Social Psychology, 78, 350-365.

Gardner, C. B., \& Gronfein, W. P. (2005). Reflections on varieties of shame induction, shame management, and shame avoidance in some works of Erving Goffman. Symbolic Interaction, 28, 175-182. 
Gilbert, P. (1998). What is shame? Some core issues and controversies. In P. Gilbert, \& B. Andrews (Eds.), Shame: Interpersonal behaviour, psychopathology and culture (pp. 3-36). New York: Oxford University Press.

Gilbert, P. (2000). The relationship of shame, social anxiety and depression: The role of the evaluation of social rank. Clinical Psychology and Psychotherapy, 7, 174-189.

Gilbert, P. (2010). Compassion focused therapy. W. Dryden (Ed.). New York: Routledge.

Gilbert, P., \& Irons, C. (2004). A pilot exploration of the use of compassionate images in a group of self-critical people. Memory, 12, 507-516.

Gilbert, P., McEwan, K., Bellew, R., Mills, A., \& Gale, C. (2009). The dark side of competition: How competitive behaviour and striving to avoid inferiority are linked to depression, anxiety, stress and self-harm. Psychology and Psychotherapy: Theory, Research and Practice, 82, 123-136.

Gilbert, P., \& Procter, S. (2006). Compassionate mind training for people with high shame and self-criticism: Overview and pilot study of a group therapy approach. Clinical Psychology and Psychotherapy, 13, 353-379.

Gomez, R., \& Leal, I. (2008). Ajustamento conjugal: características psicométricas da versão portuguesa da Dyadic Adjustment Scale. Análise Psicológica, XXVI, 625-638.

Goss, K., Gilbert, P., \& Allan, S. (1994). An exploration of shame measures: I. The "Other as Shamer Scale". Personality and Individual Differences, 17, 713-717.

Gross, C. A., \& Hansen, N. E. (2000). Clarifying the experience of shame: The role of attachment style, gender, and investment in relatedness. Personality and Individual Differences, 28, 897-907.

Harper, J. M. (2011). Regulating and coping with shame. In R. Trnka, K. Balcar \& M. Kuška (Eds.). Re-constructing emotional spaces: From experience to regulation (pp. 189-206). Prague: Prague College of Psychosocial Studies Press.

Hazan, C., \& Shaver, P. (1987). Romantic love conceptualized as an attachment process. Journal of Personality and Social Psychology, 52, 511-524.

Hayes, A. F. (2013). Introduction to mediation, moderation, and conditional process analysis: A regression-based approach. New York: Guilford Press. 
Hedman, E., Ström, P., Stünkel, A., \& Mörtberg, E. (2013). Shame and guilt in social anxiety disorder: Effects of cognitive behavior therapy and association with social anxiety and depressive symptoms. PLOS One, 8, e61713.

Joel, S., MacDonald, G., \& Shimotomai, A. (2011). Conflicting pressures on romantic relationships commitment for anxiously attached individuals. Journal of Personality, 79, 51-73.

Laurenceau, J. P., Feldman Barrett, L., \& Pietromonaco, P. R. (1998). Intimacy as an interpersonal process: The importance of self-disclosure, partner disclosure, and perceived partner responsiveness in interpersonal exchanges. Journal of Personality and Social Psychology, 74, 1238-1251.

Lewis, H. B. (1971). Shame and guilt in neurosis. New York: International Universities Press.

Lombardi, R. (2007). Shame in relation to the body, sex, and death: A clinical exploration of the psychotic levels of shame. Psychoanalytic Dialogues, 17, 385-399.

Lopes, Pinto-Gouveia, J., \& Castilho, P. (2005). Portuguese version of the Others as Shamer Scale. Unpublished manuscript.

Lopez, F.G., Gover, M. R., Leskela, J., Sauer, E. M., Schirmer, L., \& Wyssmann, J. (1997). Attachment styles, shame, guilt and collaborative problem-solving orientations. Personal Relationships, 4, 187-199.

Lopez, J. L., Riggs, S. A., Pollard, S. E., \& Hook, J. N. (2011). Religious commitment, adult attachment, and marital adjustment in newly married couples. Journal of Family Psychology, 25, 301-309.

Lutwak, N., Panish, J., \& Ferrari, J. (2003). Shame and guilt: Characterological vs. behavioral self-blame and their relationship to fear of intimacy. Personality and Individual Differences, 35, 909-916.

MacDonald, J, \& Morley, I. (2001). Shame and non-disclosure: A study of the emotional isolation of people referred for psychotherapy. British Journal of Medical Psychology, 74, 1-21.

Matos, M., Pinto-Gouveia, J., \& Costa, V. (2013). Understanding the importance of attachment in shame traumatic memory relation to depression: The impact of emotion regulation processes. Clinical Psychology and Psychotherapy, 20, 149-165.

Matos, M., Pinto-Gouveia, J., \& Duarte, C. (2012). When I don't like 
myself: Portuguese version of the Internalized Shame Scale. The Spanish Journal of Psychology, 15, 1411-1423.

Matos, M., Pinto-Gouveia, J., \& Gilbert, P. (2013). The effect of shame and shame memories on paranoid ideation and social anxiety. Clinical Psychology and Psychotherapy, 20, 334-49.

McCarthy, G., \& Maughan, B. (2010). Negative childhood experiences and adult love relationships: The role of internal working models of attachment. Attachment and Human Development, 12, 445-461.

Meyers, S. A., \& Landsberger, S. A. (2002). Direct and indirect pathways between adult attachment style and marital satisfaction. Personal Relationships, 9, 159-172.

Mikulincer, M., Florian, V., Cowan, P. A., \& Cowan, C. P. (2002). Attachment security in couple relationships: A systemic model and its implications for family dynamics. Family Process, 41, 405-434.

Mikulincer, M., Florian, V., \& Tolmacz, R. (1990). Attachment styles and fear of personal death: A case study of affect regulation. Journal of Personality and Social Psychology, 28, 273-280.

Mikulincer, M., Florian, V., \& Weller, A. (1993). Attachment styles, coping strategies and posttraumatic psychological distress: The impact of the Gulf War in Israel. Journal of Personality and Social Psychology, 64, 817-826.

Mikulincer, M., \& Orbach, I. (1995). Attachment styles and repressive defensiveness: The accessibility and architecture of affective memories. Journal of Personality and Social Psychology, 68, 917-925.

Mills, R. S. L. (2005). Taking stock of the developmental literature on shame. Developmental Review, 25, 26-63.

Murray, S. L., Bellavia, G. M., Rose, P., \& Griffin, D. W. (2003). Once hurt, twice hurtful: How perceived regard regulates daily marital interactions. Journal of Personality and Social Psychology, 84, 126-147.

Murray, S. L., Holmes, J. G., Griffin, D. W., Bellavia, G., \& Rose, P. (2001). The mismeasure of love: How self-doubt contaminates relationship beliefs. Personality and Social Psychology Bulletin, 27, 423-436.

Nathanson, D. L. (1992). Shame and pride: Affect, sex, and the birth of the self. New York: W. W. Norton.

Overall, N. C., \& Fletcher, G. J. O. (2010). Perceiving regulation from intimate partners: Reflected appraisal and self-regulation processes in close 
relationships. Personal Relationships, 17, 433-456.

Pepping, C. A., \& Halford, W. K. (2012). Attachment and relationship satisfaction in expectant first-time parents: The mediating role of relationship enhancing behaviours. Journal of Research in Personality, 46, 770-774.

Pereira, M., Canavarro, M. C., \& Narciso, I. (2009). Escala de Ajustamento Diádico Revista. Versão Portuguesa experimental (unpublished manuscript).

Pinto-Gouveia, J., \& Matos, M. (2011). Can shame memories become a key to identity? The centrality of shame memories predicts psycopathology. Applied Cognitive Psychology, 25, 281-290.

Saavedra, M. C., Chapman, K. E. \& Rogge, R. D. (2010). Clarifying links between attachment and relationship quality: Hostile conflict and mindfulness moderators. Journal of Family Psychology, 24, 380-390.

Schore, A. N. (1994). Affect regulation and the origin of the self: The neurobiology of emotional development. Hillsdale: Erlbaum.

Schore, A. N. (1996). The experience-dependent maturation of a regulatory system in the orbital prefrontal cortex and the origin of developmental psychopathology. Development and Psychopathology, 8, 59-87.

Selcuk, E., Zayas, V., \& Hazan, C. (2010). Beyond satisfaction: The role of attachment in marital functioning. Journal of Family Theory \& Review, 2, 258-279.

Stackert, R. A., \& Bursik, K. (2003). Why am I unsatisfied? Adult attachment style, gendered irrational relationship beliefs, and young adult romantic relationship satisfaction. Personality and Individual Differences, 34, 1419-1429.

Tangney, J., \& Dearing, R. (2002). Shame and guilt. New York: Guilford Press.

Treboux, D., Crowell, J. A., \& Waters, E. (2004). When "new" meets "old": Configurations of adult attachment representations and their implications for marital functioning. Developmental Psychology, 40, 295-314.

Troop, N. A., Allan, S., Serpell, L., \& Treasure, J. L. (2008). Shame in women with a history of eating disorders. European Eating Disorders Review, $16,480-488$.

Ward, P. J., Lundberg, N. R., Zabriskie, R. B., \& Barrett, K. (2009). Measuring marital satisfaction: A comparison of the Revised Dyadic 
Adjustment Scale and the Satisfaction with Married Life Scale. Marriage \& Family Review, 45, 412-429.

Williamson, I., Sandage, S. J., \& Lee, R. M. (2007). How social connectedness affects guilt and shame: Mediation by hope and differentiation of self. Personality and Individual Differences, 43, 2159-2170. 


\section{Conclusões}

Neste trabalho foi analisado o possível papel mediador da vergonha interna e externa na associação entre a vinculação e o ajustamento diádico. Tal como já referido, embora esta associação esteja já bem documentada, a informação relativa aos mecanismos subjacentes é ainda insuficiente. No entanto, este é um aspecto que merece investigação, nomeadamente porque se tem vindo a observar um aumento nas taxas de divórcio, que também se tem tornado mais precoce. A identificação de factores que podem dificultar ou comprometer as relações amorosas assume uma elevada importância para o desenho de novas estratégias de intervenção dirigidas para uma melhoria do funcionamento diádico, bem como para um aumento da satisfação e qualidade relacional.

Os resultados apresentados nesta tese demonstram que a vergonha, particularmente a vergonha interna, pode constituir um importante mediador entre a ansiedade e o evitamento de vinculação e o ajustamento diádico. A identificação deste factor é importante já que corresponde a um processo psicológico que pode ser trabalhado com sucesso em terapia, nomeadamente através da terapia focada na compaixão, ao contrário do que acontece com as orientações de vinculação. Tal como já descrito, as experiências precoces com as figuras de vinculação são estruturadas em esquemas interpessoais (os modelos dinâmicos internos do $\mathrm{Eu}$ e dos Outros), que, como qualquer outro esquema nuclear, não estão facilmente acessíveis a intervenção e são resistentes a mudança. Embora se considere uma relativa estabilidade dos modelos dinâmicos internos, também se aceita que estes podem ser revistos, ao longo da vida, nomeadamente através de experiências positivas, desconfirmatórias, com outros significativos (Feeney, 2008; Treboux e tal., 2004). Neste contexto, os parceiros amorosos assumem particular importância. Assim, uma das formas de promover a revisão dos esquemas interpessoais será a promoção de relações mais satisfatórias e gratificantes, o que pode ser conseguido através do fornecimento de novas ferramentas para o indivíduo lidar com os desafios quotidianos das relações íntimas.

No entanto, o que os nossos resultados sugerem, em conjunto com dados descritos na literatura, é que alguns desses desafios (e.g. dificuldades de autorevelação, comunicacionais e de gestão de conflitos) podem ter subjacentes 
aspectos mais nucleares do Eu, como a vergonha, que devem ser equacionados no contexto terapêutico. Por exemplo, a vergonha, bem como o medo do impacto nos outros da revelação e expressão emocional que lhe está associado, pode conduzir ao evitamento da auto-revelação e honestidade nas relações, o que, por sua vez, pode levar ao acumular de ressentimentos que vão minar a relação (Gilbert, 2010). Como tal, a adopção de estratégias submissas e o esconder de sentimentos podem ter efeitos perversos conduzindo aos efeitos indesejados e temidos: a rejeição, o fim da relação. Consequentemente, a pessoa conclui que não é amável, atribuindo a rejeição a características do Eu, e não reconhece que são os seus comportamentos de segurança que estão a causar o problema (Gilbert, 2010).

Gilbert (2010), no seu modelo tripartido da regulação emocional, propôs a existência de três sistemas evolutivos: o sistema afiliativo, o sistema de ameaça/defesa e o sistema de drive. O sistema de vinculação tem um grande impacto nestes sistemas. Experiências relacionais precoces negativas (vinculação insegura), nomeadamente as geradoras de vergonha, levam ao sobre-desenvolvimento do sistema de ameaça/defesa e ao sub-desenvolvimento do sistema afiliativo. Este último sistema, activado por sinais de cuidado e afiliação, é muito importante para o bem estar físico e psicológico dos seres humanos, pois gera sentimentos de calor, segurança e pertença, e regula os sistemas de ameaça e de drive. Adicionalmente, experiências precoces negativas com os cuidadores primários podem fazer com que o sistema afiliativo e a necessidade de tranquilização fiquem alternativamente associados a tristeza, sofrimento, ameaça ou punição. Desta forma, as memórias de vergonha, quando são reactivadas, não só desencadeiam sentimentos de medo e de vergonha, mas também de tristeza e solidão, comprometendo a regulação do sistema de ameaça-defesa, já que o acesso ao sistema afiliativo fica bloqueado (Gilbert, 2010). Assim, um dos objectivos terapêuticos de trabalhar a vergonha e as memórias de vergonha será promover a activação do sistema de afiliação, de forma a que as pessoas experimentem calor, segurança e ligação nas relações íntimas. Complementarmente, esta aproximação terapêutica resultará também numa maior regulação da activação do sistema de ameaça (Gilbert, 2010; Gilbert \& Procter, 2006).

A capacidade do indivíduo para se sentir seguro no mundo social e nas relações tem muitas vezes origem na forma como este sente ou pensa que os 
outros o vêem, o que está intimamente ligado ao modo como o indivíduo se vê a si próprio (Gilbert, 2010). Esta capacidade é crucial para a regulação emocional. Se o indivíduo for capaz de criar na mente dos outros sentimentos de valorização e uma visão positiva de si, o mundo envolvente torna-se mais seguro, dado que o indivíduo não tem medo de ser rejeitado ou atacado (porque o outro o valoriza), conseguindo co-criar papéis significativos de apoio mútuo, relações de carácter sexual e partilha íntima. A recepção de sinais de valorização e cuidados por parte de outros significativos tem também efeitos directos a nível do sistema afiliativo e respostas neuroendócrinas associadas (Gilbert, 2010). Adicionalmente, a revisão do significado autobiográfico associado às memórias de vergonha pode contribuir para a reavaliação e reestruturação dos modelos dinâmicos internos do Eu e dos Outros (Matos et al, 2013; Davila \& Cobb, 2004, cit. in Feeney, 2008).

Ajudar o indivíduo a lidar com a vergonha de forma mais autocompassiva e adaptativa poderá, então, contribuir para um melhor funcionamento interpessoal e para uma vivência mais satisfatória e gratificante das relações amorosas.

\section{Bibliografia}

Feeney, J. A. (2008). Adult romantic attachment: Developments in the study of couple relationships. In J. Cassidy \& P. R. Shaver (Eds.), Handbook of attachment: Theory, research and clinical applications ( ${ }^{\text {nd }}$ Ed.; pp. 456-481). New York: Guilford Press.

Gilbert, P. (2010). Compassion focused therapy. W. Dryden (Ed.). New York: Routledge.

Gilbert, P., \& Procter, S. (2006). Compassionate mind training for people with high shame and self-criticism: Overview and pilot study of a group therapy approach. Clinical Psychology and Psychotherapy, 13, 353-379.

Matos, M., Pinto-Gouveia, J., \& Costa, V. (2013). Understanding the importance of attachment in shame traumatic memory relation to depression: The impact of emotion regulation processes. Clinical Psychology and Psychotherapy, 20, 149-165.

Treboux, D., Crowell, J. A., \& Waters, E. (2004). When "new" meets "old": Configurations of adult attachment representations and their implications for marital functioning. Developmental Psychology, 40, 295-314. 